\title{
NUMERICAL STUDY OF TURBULENT MIXING LAYERS WITH NON-EQUILIBRIUM IONIZATION CALCULATIONS
}

\author{
Kyujin KwaK and Robin L. Shelton \\ Department of Physics and Astronomy, University of Georgia, Athens, GA 30602, USA \\ Received 2010 March 23; accepted 2010 June 15; published 2010 July 21
}

\begin{abstract}
Highly ionized species, such as $\mathrm{CIV}, \mathrm{Nv}$, and $\mathrm{O}$ VI, are commonly observed in diffuse gas in various places in the universe, such as in our Galaxy's disk and halo, high velocity clouds (HVCs), external galaxies, and the intergalactic medium. These ions are often used to trace hot gas whose temperature is a few times $10^{5} \mathrm{~K}$. One possible mechanism for producing high ions is turbulent mixing of cool gas (such as that in a high or intermediate velocity cloud) with hotter (a few times $10^{6} \mathrm{~K}$ ) gas in locations where these gases slide past each other. By using hydrodynamic simulations with radiative cooling and non-equilibrium ionization (NEI) calculations, we investigate the physical properties of turbulent mixing layers and the production of high ions ( $\mathrm{CIV}, \mathrm{Nv}$, and $\mathrm{O} \mathrm{VI})$. We find that most of the mixing occurs on the hot side of the hot/cool interface, where denser cool gas is entrained and mixed into the hotter, more diffuse gas. Our simulations reveal that the mixed region separates into a tepid zone containing radiatively cooled, CiV-rich gas and a hotter zone which is rich in $\mathrm{C}$ IV, $\mathrm{NV}$, and $\mathrm{O}$ vi. The hotter zone contains a mixture of low and intermediate ions contributed by the cool gas and intermediate and high-stage ions contributed by the hot gas. Mixing occurs faster than ionization or recombination, making the mixed gas a better source of $\mathrm{CIV}, \mathrm{Nv}$, and $\mathrm{OVI}$ in our NEI simulations than in our collisional ionization equilibrium (CIE) simulations. In addition, the gas radiatively cools faster than the ions recombine, which also allows large numbers of $\mathrm{C} I V, \mathrm{NV}$, and $\mathrm{O}$ VI ions to linger in the NEI simulations. For these reasons, our NEI calculations predict more $\mathrm{C}$ IV, $\mathrm{Nv}$, and $\mathrm{O}$ vi than our CIE calculations predict. We also simulate various initial configurations and find that more CIV is produced when the shear speed is smaller or the hot gas has a higher temperature. We find no significant differences between simulations having different perturbation amplitudes in the initial boundary between the hot and cool gas. We discuss the results of our simulations, compare them with observations of the Galactic halo and highly ionized HVCs, and compare them with other models, including other turbulent mixing calculations. The ratios of $\mathrm{C}$ IV to $\mathrm{NV}$ and $\mathrm{NV}$ to $\mathrm{O}$ VI are in reasonable agreement with the averages calculated from observations of the halo. There is a great deal of variation from sightline to sightline and with time in our simulations. Such spatial and temporal variation may explain some of the variation seen among observations.
\end{abstract}

Key words: Galaxy: halo - hydrodynamics - methods: numerical - turbulence - ultraviolet: ISM

Online-only material: color figures

\section{INTRODUCTION}

Highly ionized species such as $\mathrm{C}$ IV, $\mathrm{N} \mathrm{v}$, and $\mathrm{O}$ vi are often used as tracers for diffuse gas within the temperature range of $(1-3) \times 10^{5} \mathrm{~K}$. These high ions are found by absorption line measurements in various places in the universe, including the disk (Bowen et al. 2008; Sallmen et al. 2008; Savage et al. 2001a; Savage \& Massa 1987; Cowie et al. 1981; Jenkins 1978a, 1978b) and the halo of the Milky Way (Ganguly et al. 2005; Savage et al. 2001b, 2003; Zsargó et al. 2003; Sterling et al. 2002; Savage et al. 1997; Sembach et al. 1997; Savage \& Sembach 1994; Sembach \& Savage 1992). The emission lines of these high ions are also found in the Galaxy (Shelton et al. 2001, 2007; Dixon et al. 2006; Otte \& Dixon 2006; Korpela et al. 2006; Welsh et al. 2007). Most of the observed high ions in the Galactic disk and halo are nearly stationary.

There is also a population of high ions moving at velocities of a few hundreds of $\mathrm{km} \mathrm{s}^{-1}$ with respect to the local standard of rest (LSR; Collins et al. 2007; Fox et al. 2005, 2004; Sembach et al. 2003). The distances to some of these ions are unknown. Most of these ions are on sightlines that intersect $\mathrm{HI}$ high velocity clouds (HVCs), but some are not. Thus, these latter ions are due to ionized HVCs.

High ions are also found in external galaxies via absorption line measurements (LMC; Lehner \& Howk 2007;
Danforth \& Blair 2006; Sankrit et al. 2004) and O VI emission measurements (Bregman et al. 2006b; Ganguly et al. 2006). High ions are detected not only in nearby galaxies but also in damped Ly $\alpha$ systems for distant galaxies (Fox et al. 2009, 2007) and gamma-ray burst (GRB) host galaxies (Prochaska et al. 2008; Fox et al. 2008). Even the intergalactic medium contains high ions. In the nearby intergalactic space, high ions are detected along sightlines to distant QSOs (Tripp et al. 2008). In the cooling flows of clusters of galaxies such as Abell 426, Abell 1795, and Abell 2597, high ions are observed in the flow gas cooling from hotter, X-ray-emitting gas (Bregman et al. 2006a; Oegerle et al. 2001; Dixon et al. 1996).

Observations of $\mathrm{CIV}, \mathrm{NV}$, and $\mathrm{O} v \mathrm{v}$ in various places in the universe indicate that these ions are very commonly produced. How they are produced and what their existence implies about the local physical conditions have been longstanding questions. For example, in order to trace the detailed physical condition of the plasma in the Galactic disk and halo, ratios between the quantities of different ions, either their absorption column density or their emission intensity, are measured and compared with various model predictions (Gnat \& Sternberg 2007; Indebetouw \& Shull 2004a, 2004b; Shull \& Slavin 1994; Slavin et al. 1993; Slavin \& Cox 1992; Borkowski et al. 1990; Edgar \& Chevalier 1986). 
One of several possible production mechanisms for the high ions is turbulent mixing in places where hot and cool gas flow past each other. This idea is supported by the fact that the universe is also rich in hot, X-ray-emitting gas and that the regions containing high ions are sometimes correlated with hotter, X-ray-emitting regions. Early analytic models of mixing layers were made by Begelman \& Fabian (1990). They estimated the temperature of the mixed layer as the geometrical density-weighted mean of the hot and cool gas temperatures under the assumption that two gases mix due to turbulence and that mixing is efficient. Slavin et al. (1993) further developed Begelman \& Fabian's (1990) idea and analytically calculated the emission spectra and column densities of high ions under the assumption that mixing reaches steady state. Their calculated ratios between the quantities of different ions are often used as diagnostics for observations. The results of Slavin et al. (1993) were tested by Esquivel et al. (2006) who used threedimensional magnetohydrodynamic (MHD) simulations. They assumed collisional ionization equilibrium (CIE) for their column density calculations. They simulated the mixing layer on a $10 \mathrm{pc}$ wide computational domain for durations up to $3 \mathrm{Myr}$, but they found that their simulations did not reach steady state as Slavin et al. (1993) had assumed. Although their calculated ion ratios can differ significantly with time and sightline, their ratios are more similar to the analytic results of Slavin et al. (1993) than the average ratios calculated for other phenomena such as radiative cooling.

The next logical step in the progression is to calculate the ion content using non-equilibrium ionization (NEI) algorithms. In this paper, we do that. We run detailed hydrodynamic simulations that include NEI calculations of interesting ions. We also perform CIE calculations for comparison. In order to offset the memory demands of the NEI calculations, we reduce the spatial dimensions from three to two. Our NEI results provide better predictions for high ion column densities and ion ratios and can be used as updated diagnostics for comparison with observations. Our simulations reveal that NEI calculations predict more high ions than CIE calculations, although the ion ratios do not change dramatically. We examine the reason for the difference between NEI and CIE by looking into the distribution of ionization levels of interesting atoms (Section 3.1.3).

We also consider various physical conditions that form turbulent mixing layers and find that the detailed configuration of the mixing layer, such as the perturbation amplitude of the initial boundary between the hot and cool gas, does not affect the ion ratios as long as mixing is efficient. However, the initial velocity difference between the hot and the cool gas and the temperature of the hot gas affect the ion ratios. When the initial speed has a slower value $\left(50 \mathrm{~km} \mathrm{~s}^{-1}\right)$ and the hot gas has a higher temperature $\left(3 \times 10^{6} \mathrm{~K}\right)$, the ion ratios show different behavior than when the initial speed is $100 \mathrm{~km} \mathrm{~s}^{-1}$ and the hot gas is $1 \times 10^{6} \mathrm{~K}$ in temperature. These trends may be used as diagnostics that shed light on the conditions of the mixing gas (Section 3.4.4).

When compared with analytic turbulent mixing models by Slavin et al. (1993), our model simulations both from NEI and CIE calculations produce somewhat smaller ratios of $\mathrm{C}$ IV column density to $\mathrm{O}$ vi column density, $N(\mathrm{C}$ IV $) / N(\mathrm{O}$ vI), and/or somewhat larger $N(\mathrm{~N} \mathrm{v}) / N(\mathrm{O} v \mathrm{vI})$ ratios. Our ion ratios are more similar to the CIE simulations done by Esquivel et al. (2006) in which radiative cooling was allowed. We find that our average simulated ion ratios are close to the average values from halo observations. Individual observations can vary greatly from the average. Some of the variation in the observed ratios may be due to line of sight geometry or age of the mixing layer, given that the ion ratios calculated for individual sightlines in our domain vary greatly with time and sightline location. We also compare our simulated ion ratios with those observed in Complex $\mathrm{C}$ and find that our model simulations (and other turbulent mixing models) are more likely to produce the observed high ion ratios in Complex $\mathrm{C}$ when the low metallicity of Complex $\mathrm{C}$ is considered.

This paper is organized as follows. The next section provides the numerical methods and physical parameters used in our simulations. Section 3 explains the results of our numerical study and Section 4 compares them with observations of the halo and Complex C. Section 5 presents the summary.

\section{NUMERICAL METHODS AND PHYSICAL PARAMETERS}

We use FLASH version 2.5 for our simulations (Fryxell et al. 2000). We include radiative cooling by using relevant modules in FLASH. We also use the FLASH NEI module to track the degree of ionization of the carbon, nitrogen, and oxygen atoms in the gas in each zone in a time-dependent fashion. In FLASH, each time step's NEI calculation is done in two pieces. In the first piece, which is done as a part of the hydrodynamic update, the mass density of each ion is updated via the mass conservation equation. During the hydrodynamic update, the total mass density and temperature are also updated. They will be used as inputs for the second piece of the NEI calculation. In the second piece, which occurs after the hydrodynamic update, the populations of the ionization levels within the atoms (of carbon, nitrogen, and oxygen) are updated by solving sets of ordinary differential equations which include the ionization and recombination rates for each ion. In FLASH, the ionization and recombination rates include the effects of collisional ionization, auto-ionization, radiative recombination, and dielectronic recombination.

In the FLASH NEI calculations, we set the abundances of carbon, nitrogen, and oxygen to be consistent with those used in the Raymond and Smith code (Raymond \& Smith 1977; downloaded from HEASARC), which is used for the CIE calculations. In our CIE calculations, the Raymond and Smith code calculates the populations of the ionization levels in the atoms in each zone as a function of the zone temperature reported by the hydrodynamic simulations. The ionization fractions multiplied by the elemental abundance and the volume density reported by the hydrodynamic code yield the volume densities of ions for each zone. The column densities are obtained by integrating the volume densities along sightlines.

It is worth mentioning that our calculations are approximate because we do not calculate the effects of NEI ionization levels on the radiative energy loss rate. Calculating the total radiative loss rate from the sum of the loss rates from individual NEI ions would require very large computing resources. Instead, in our simulations, we use the CIE cooling curve calculated from the $\mathrm{CIE}$ cooling rates of all of the relevant elements. Assuming that the plasma radiatively cools according to the CIE cooling curve at each time step of the simulation allows us to save significant computing resources.

In order to verify the validity of this approximation, more complete future studies would be required, which compare simulations using NEI cooling with simulations using CIE cooling. The currently available comparison studies between 


\begin{tabular}{|c|c|c|c|c|c|c|c|c|c|c|}
\hline & & & & & $\begin{array}{l}\text { Table } \\
\text { Mode }\end{array}$ & & & & & \\
\hline \multirow[t]{2}{*}{ Model } & \multicolumn{2}{|c|}{ Domain } & \multirow{2}{*}{$\begin{array}{l}\text { Maximum } \\
\text { Refinement } \\
\text { Level }\end{array}$} & \multicolumn{3}{|c|}{ Hot Gas } & \multirow{2}{*}{$\begin{array}{l}\text { Initial Interface } \\
\qquad y=f(x)\end{array}$} & \multicolumn{3}{|c|}{ Cool Gas } \\
\hline & $\begin{array}{c}x \\
(\mathrm{pc})\end{array}$ & $\begin{array}{c}y \\
(\mathrm{pc})\end{array}$ & & $\begin{array}{c}n_{H} \\
\left(\mathrm{~cm}^{-3}\right)\end{array}$ & $\begin{array}{c}T \\
(\mathrm{~K})\end{array}$ & $\begin{array}{c}v_{x} \\
\left(\mathrm{~km} \mathrm{~s}^{-1}\right)\end{array}$ & & $\begin{array}{c}n_{H} \\
\left(\mathrm{~cm}^{-3}\right)\end{array}$ & $\begin{array}{c}T \\
(\mathrm{~K})\end{array}$ & $\begin{array}{c}v_{x} \\
\left(\mathrm{~km} \mathrm{~s}^{-1}\right)\end{array}$ \\
\hline $\mathrm{A}^{\mathrm{a}}$ & {$[0,100]$} & {$[-250,50]$} & 6 & $10^{-4}$ & $10^{6}$ & 0 & $y=(2.5 \mathrm{pc}) \sin \left(\frac{2 \pi x}{100 \mathrm{pc}}\right)$ & 0.1 & $10^{3}$ & 100 \\
\hline B & {$[0,100]$} & {$[-250,50]$} & $7^{\mathrm{b}}$ & $10^{-4}$ & $10^{6}$ & 0 & $y=(2.5 \mathrm{pc}) \sin \left(\frac{2 \pi x}{100 \mathrm{pc}}\right)$ & 0.1 & $10^{3}$ & 100 \\
\hline $\mathrm{C}$ & {$[0,10]^{\mathrm{c}}$} & {$[-25,5]$} & 6 & $10^{-4}$ & $10^{6}$ & 0 & $y=(0.25 \mathrm{pc}) \sin \left(\frac{2 \pi x}{10 \mathrm{pc}}\right)$ & 0.1 & $10^{3}$ & 100 \\
\hline $\mathrm{D}$ & {$[0,100]$} & {$[-250,50]$} & 6 & $10^{-4}$ & $10^{6}$ & 0 & $y=(2.5 \mathrm{pc}) \sin \left(\frac{2 \pi x}{100 \mathrm{pc}}\right)$ & 0.1 & $10^{3}$ & $50^{\mathrm{d}}$ \\
\hline $\mathrm{E}$ & {$[0,100]$} & {$[-250,50]$} & 6 & $10^{-4}$ & $10^{6}$ & 0 & $y=(5.0 \mathrm{pc}) \sin \left(\frac{2 \pi x}{100 \mathrm{pc}}\right)^{\mathrm{e}}$ & 0.1 & $10^{3}$ & 100 \\
\hline $\mathrm{F}$ & {$[0,100]$} & {$[-250,50]$} & 6 & $\frac{1}{3} \times 10^{-4}$ & $3 \times 10^{6 f}$ & 0 & $y=(2.5 \mathrm{pc}) \sin \left(\frac{2 \pi x}{100 \mathrm{pc}}\right)$ & 0.1 & $10^{3}$ & 100 \\
\hline
\end{tabular}

\section{Notes.}

${ }^{a}$ Reference simulation.

${ }^{\mathrm{b}}$ Higher spatial resolution by one refinement level (factor 2) than Model A.

${ }^{\mathrm{c}}$ The computational domain is $1 / 10$ of Model A.

${ }^{\mathrm{d}}$ Cool gas has half initial speed of Model A.

${ }^{\mathrm{e}}$ Amplitude of initial interface between hot and cool gas is twice that of Model A.

$\mathrm{f}$ The temperature of the hot gas is three million Kelvin.

CIE and NEI cooling rates show opposite trends depending upon whether the gas is in the process of ionizing (because its temperature has been raised) or is in the process of recombining (because its temperature has fallen). Sutherland \& Dopita (1993) and Gnat \& Sternberg (2007) calculated the NEI radiation rates from all relevant ions (as well as their ionization states) for cooling gas. Both studies showed that the NEI cooling rates are lower than the CIE cooling rates because the recombination of high-stage ions in the cooling gas is delayed. In contrast, cool gas in the process of heating due to external heat sources shows delayed ionization and appears to have NEI cooling rates that are higher than the CIE cooling rate (Gnat et al. 2010). In turbulent mixing layers, we can see both delayed recombination and delayed ionization in the mixed gas (this is verified by our simulations; see Section 3.1.3). Thus, the true cooling rate would be a complex combination of rates that sometimes exceed and sometimes fall below the CIE rate.

We run our simulations in two-dimensional Cartesian coordinates for two reasons. First, the previous three-dimensional MHD study of Esquivel et al. (2006) showed that a 10 pc scale during $3 \mathrm{Myr}$ was not long enough to see efficient mixing of two gases. So, it is necessary to run larger-scale simulations to a later time with significant spatial resolution. The mixing zone expands over time, so long duration runs must also have larger domains. Second, tracing ion fractions of carbon, nitrogen, and oxygen requires more computing resources. We find that twodimensional hydrodynamic simulations with $100 \mathrm{pc} \times 300 \mathrm{pc}$ domains running for several tens of Myr reveal the physical properties of high ions in mixing layers. In order to check the validity of our simulations, we also run a simulation with a $10 \mathrm{pc}$ wide domain for $8 \mathrm{Myr}$.

In this paper, we present the results of six simulations, labeled Models A, B, C, D, E, and F. Model A is our reference simulation; each of the other models is made after varying one of Model A's parameters and runs in order to test the effect of that parameter. In Model A, the computational domain is $100 \mathrm{pc} \times$ $300 \mathrm{pc}$ and the maximum refinement level is 6 . According to the FLASH adaptive mesh refinement convention, this means that if our grid were fully refined, it would have $256 \times 768$ zones and the smallest zone would be $(0.4 \mathrm{pc})^{2}$. At the beginning of the simulation, the hot gas $\left(n_{H}=10^{-4} \mathrm{~cm}^{-3}, T=10^{6} \mathrm{~K}\right)$ occupies the lower $5 / 6$ of the domain and the cool gas $\left(n_{H}=0.1 \mathrm{~cm}^{-3}\right.$, $T=10^{3} \mathrm{~K}$ ) occupies the remainder. The gas is initially in pressure balance with $P / k_{B}=230 \mathrm{~cm}^{-3} \mathrm{~K}$ (considering that the number of helium atoms is $10 \%$ of that of hydrogen atoms). Note that this low thermal pressure is characteristic of the halo but the thermal pressure in the Galactic disk is higher by at least an order of magnitude (Ferriere 1998; Jenkins 2004; Cox 2005). However, as Slavin et al. (1993) pointed out, the column densities are independent of the thermal pressure although the emission intensity is proportional to the thermal pressure. The thermal pressure would also affect the speed at which the turbulent mixing layers develop.

In order to create shear, the cool gas moves to the right (toward the positive $x$-direction) at $100 \mathrm{~km} \mathrm{~s}^{-1}$ throughout the simulation while the hot gas does not. Periodic boundary conditions on the left and right sides and outflow boundary conditions on the top and bottom sides enable such motion. In order to seed the turbulence, we add curvature to the initial boundary between the cool gas and the hot gas. The shape of our boundary is $y=(2.5 \mathrm{pc}) \sin \left(\frac{2 \pi x}{100 \mathrm{pc}}\right)$.

Model B is a higher spatial resolution analog of Model A. It has a maximum refinement level of 7 , which would correspond to $512 \times 1536$ zones if the domain were fully resolved. Model C has a smaller computational domain (1/10 in height and width), Model D has a smaller initial speed difference $\left(50 \mathrm{~km} \mathrm{~s}^{-1}\right)$ between the cool gas and the hot gas, Model E has a larger ripple (maximum amplitude $=5 \mathrm{pc}$ ) in the boundary between the gases, and Model $\mathrm{E}$ has hotter $\left(T=3 \times 10^{6} \mathrm{~K}\right)$ and less dense $\left(n_{H}=\frac{1}{3} \times 10^{-4} \mathrm{~cm}^{-3}\right)$ hot gas than Model A. Our model parameters are summarized in Table 1.

\section{RESULTS}

\subsection{Model A: Reference Simulation}

The sequences of snapshots of Model A presented in Figure 1 show the mixing zone between the hot and cool gas developing and growing in time. Five sequences are presented. They portray hydrogen number density, temperature, and indicators of the prevalence of $\mathrm{CIV}, \mathrm{Nv}$, and $\mathrm{O}$ VI. The first snapshot in each sequence shows the model at $t=0 \mathrm{Myr}$, when it contains only 

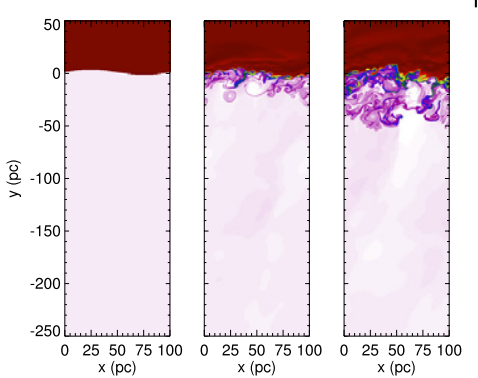

$\log _{10}$ [ Hydrogen Number Density $\left(\mathrm{cm}^{-3}\right)$ ]
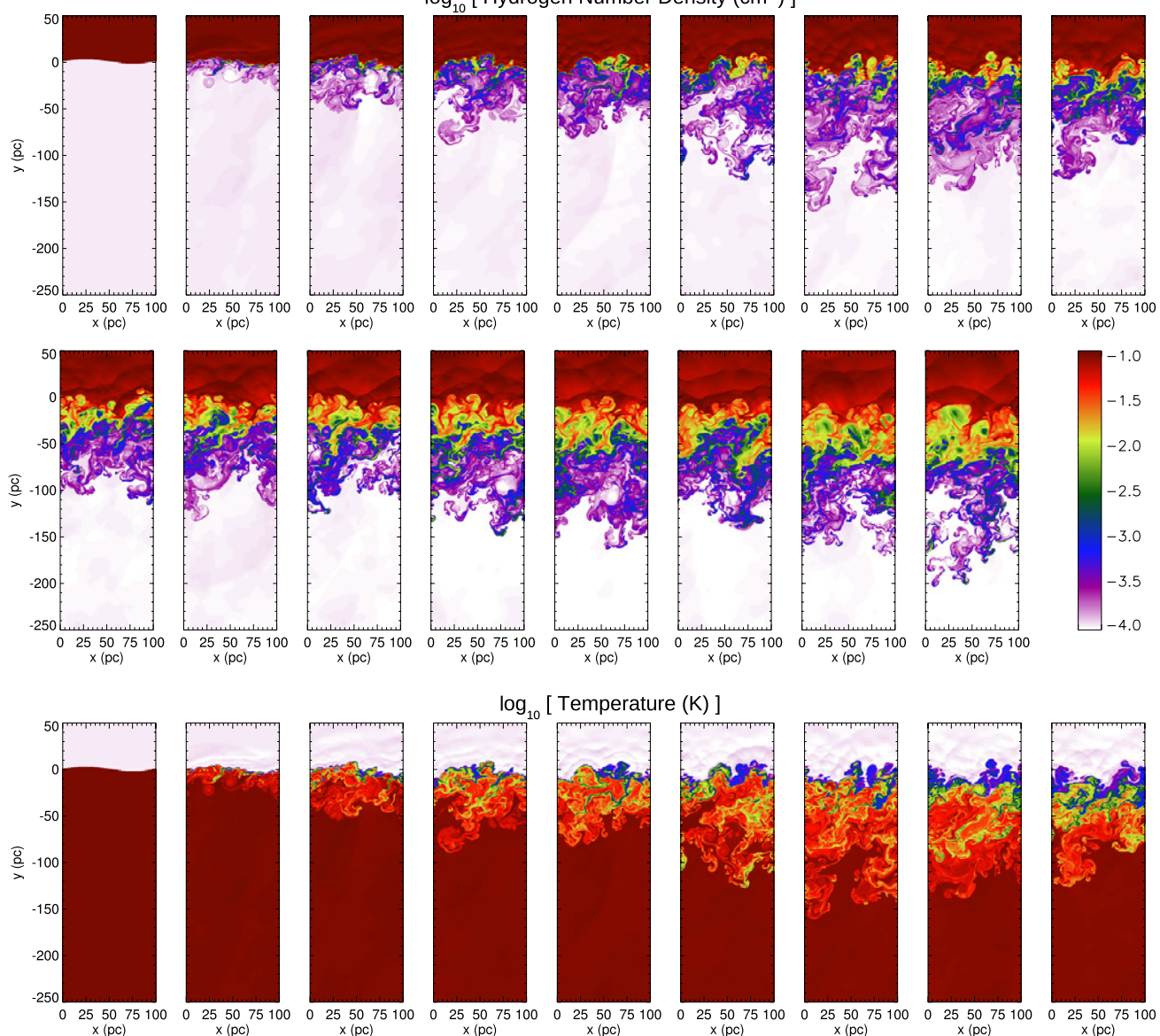

$\log _{10}[$ Temperature $(K)]$
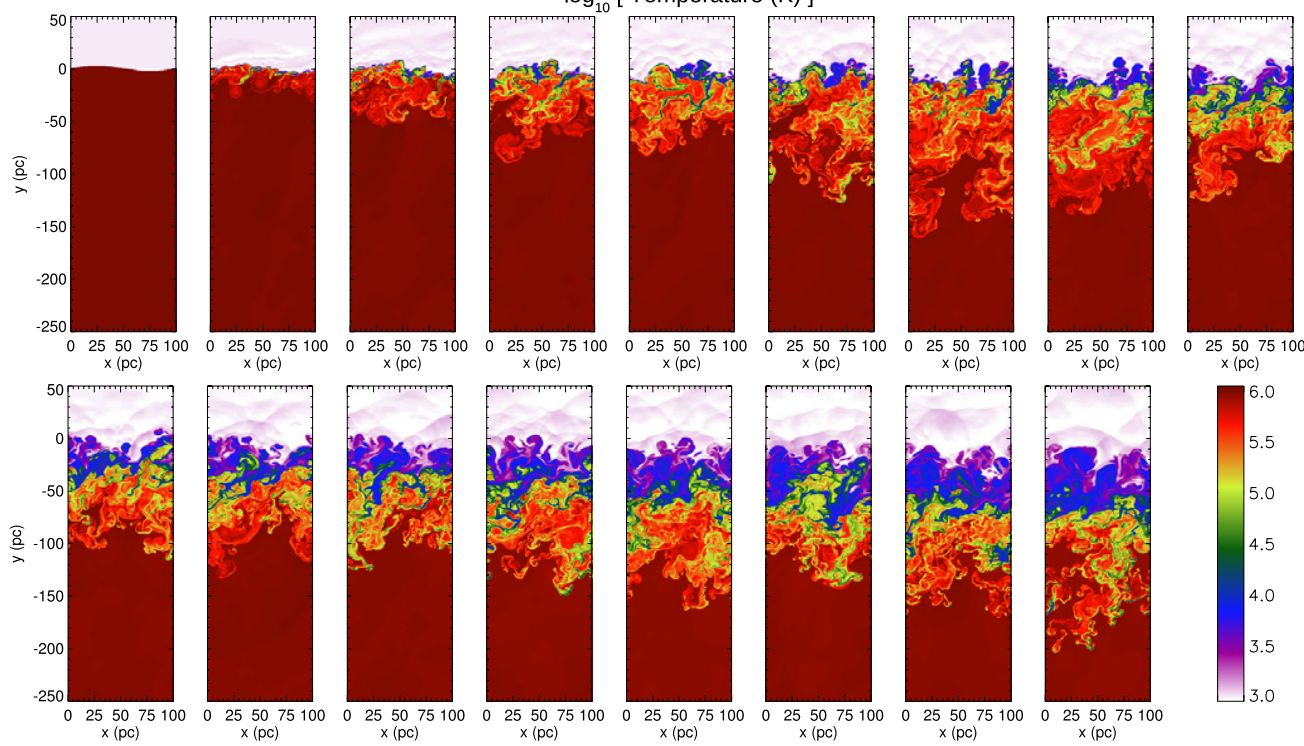

Figure 1. Model A: the top two panels depict the log of the hydrogen number density and the bottom two panels depict the log of the temperature. The leftmost plots in the first and third rows show the model at $t=0 \mathrm{Myr}$. A time period of $5 \mathrm{Myr}$ elapses between successive plots. Model A: population of C Iv (top two panels) $\mathrm{N} \mathrm{v}$ (middle two panels), and $\mathrm{O}$ VI (bottom two panels) in log scale as a function of time obtained from NEI calculations. In these plots, the population of $\mathrm{C}$ IV, $\mathrm{N}$ v, or $\mathrm{O}$ VI refers to the fraction of ions in the specified ionization stage compared to the total number of ions of carbon, nitrogen, and oxygen, respectively.

(A color version of this figure is available in the online journal.)

hot, rarefied gas in the lower portion of the grid and cool, denser gas in the upper portion of the grid. Between this epoch and $t=5 \mathrm{Myr}$ (shown as the next snapshot in each sequence), the relative motion between the hot and cool layers has stirred the gas, creating a zone of mixed, intermediate temperature gas between the hot and cool layers. Most of the mixing shown in our simulations occurs on the hot side of the hot-cool interface, where dense cool gas is entrained and mixed into stationary hot, rarefied gas. The entrained gas expands due to the raising of its temperature. Whatever hot gas becomes entrained into the cool gas compresses as its temperature falls and moves off of the grid at roughly $100 \mathrm{~km} \mathrm{~s}^{-1} \approx 100 \mathrm{pc} \mathrm{Myr}^{-1}$ and so appears only fleetingly in the simulations.

When tendrils of cool gas first begin to intrude into and mix with the hot gas, the temperature of the entrained gas rises immediately to the geometric mean. Its ionization state, however, does not equilibrate as quickly and thus the freshly entrained gas contains a mixture of poorly ionized gas that is in the process of ionizing and highly ionized gas that is in the process of recombining. As the next several snapshots (each of which is spaced $5 \mathrm{Myr}$ apart) show, the tendrils of intermediate temperature mixed gas intrude into the hot gas as time goes by, increasing the depth of the mixed zone.

The total depth of the mixed zone grows for the first $30 \mathrm{Myr}$ then shrinks slightly, then begins to grow again around $50 \mathrm{Myr}$. Because the timescale of the growth spurts is a large fraction of the simulation time, it is not possible to conclude that the mixing layer has achieved a steady state, even after 80 Myr. Note that individual cool clouds in the Galaxy should be much shorter than $8 \mathrm{kpc}$, the distance through which the hot and cool material in Model A slide past each other in 80 Myr. Thus, carrying out the simulations to larger times would not be realistic. 

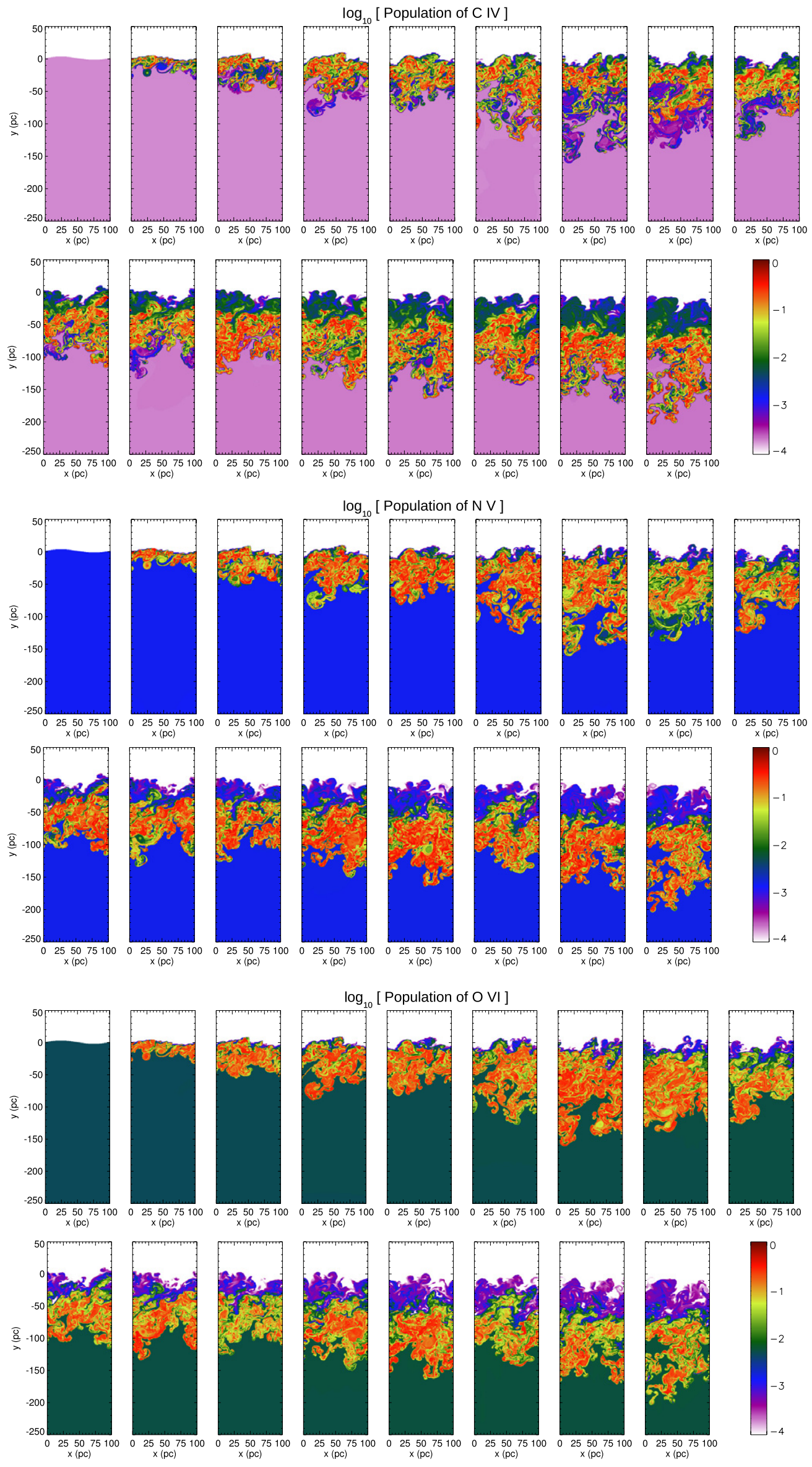

Figure 1. (Continued) 



Figure 2. Average high-stage ion column densities for all vertical sightlines in Model A (left column), Model B (middle column), and Model C (right column) as a function of time. Top panels: column densities of C IV (short dashed lines), Nv (long dashed lines), and O vi (solid lines), where the dark lines are from NEI calculations and the gray lines are from CIE calculations. Second row of panels: ratios of NEI to CIE column densities for each ion. Third to fifth rows: ratios between the column densities of different ions; here the dark lines are from the NEI calculations and the gray lines are from the CIE calculations.

Mixing plays an important role with regards to radiative cooling. Intermediate temperature $\left(T \sim 10^{5} \mathrm{~K}\right)$ mixed gas cools far faster than hotter gas of the same density. Thus, the mixing process seeds the hot gas with what it needs (cool gas) to cool quickly. Radiative cooling in the $T=1.5 \times 10^{5} \mathrm{~K}$, $n=6.8 \times 10^{-4} \mathrm{~cm}^{-3}$ mixed gas (such as that beneath the interface at $t=5 \mathrm{Myr}$ ) occurs on the timescale $t_{\mathrm{cool}}=\frac{3}{2} \frac{k T}{n \Lambda(T)} \approx$ $2 \mathrm{Myr}$ (where $\Lambda(T)$ is the cooling coefficient). As a result, this gas quickly cools to $\sim 10^{4} \mathrm{~K}$, the temperature at which cooling ceases to be applied by FLASH. As time progresses, neighboring mixed gas cools and joins it to make an ever-deepening layer of $T \sim 10^{4} \mathrm{~K}$ gas. This layer contains, but is not especially rich in, $\mathrm{C}$ IV ions. It also contains $\mathrm{N} \mathrm{v}$ and $\mathrm{O} v \mathrm{VI}$ ions, but in smaller portions to their atomic abundances. The hotter mixed gas is richer in all three of these ions.

\subsubsection{Calculation of Column Densities}

We calculate the average column densities of $\mathrm{CIV}, \mathrm{Nv}$, and $\mathrm{O}$ VI ions along vertical sightlines, i.e., parallel to the $y$-axis through the Model A domain, and plot them at $1 \mathrm{Myr}$ intervals in Figure 2. The plots present the column densities obtained using both the NEI and CIE algorithms, showing that the NEI algorithms yield much higher column densities than do 
Table 2

Column Densities along Vertical Sightlines at $t=30 \mathrm{Myr}$ in Model A

\begin{tabular}{|c|c|c|c|c|c|c|c|c|}
\hline \multirow[t]{2}{*}{ Ions } & \multicolumn{4}{|c|}{ NEI } & \multicolumn{4}{|c|}{ CIE } \\
\hline & \multicolumn{4}{|c|}{$\left(10^{12} \mathrm{~cm}^{-2}\right)$} & \multicolumn{4}{|c|}{$\left(10^{12} \mathrm{~cm}^{-2}\right)$} \\
\hline Civ & 7.69 & 7.36 & 3.31 & {$[2.73,16.17]$} & 1.56 & 1.54 & 0.81 & {$[0.13,3.87]$} \\
\hline $\mathrm{Nv}$ & 1.49 & 1.43 & 0.35 & {$[0.92,2.41]$} & 0.58 & 0.54 & 0.24 & {$[0.17,1.32]$} \\
\hline O VI & 9.33 & 9.29 & 1.47 & {$[6.08,12.19]$} & 4.18 & 4.10 & 0.80 & {$[2.33,6.98]$} \\
\hline
\end{tabular}

Note.

${ }^{\text {a }}$ Standard deviation.

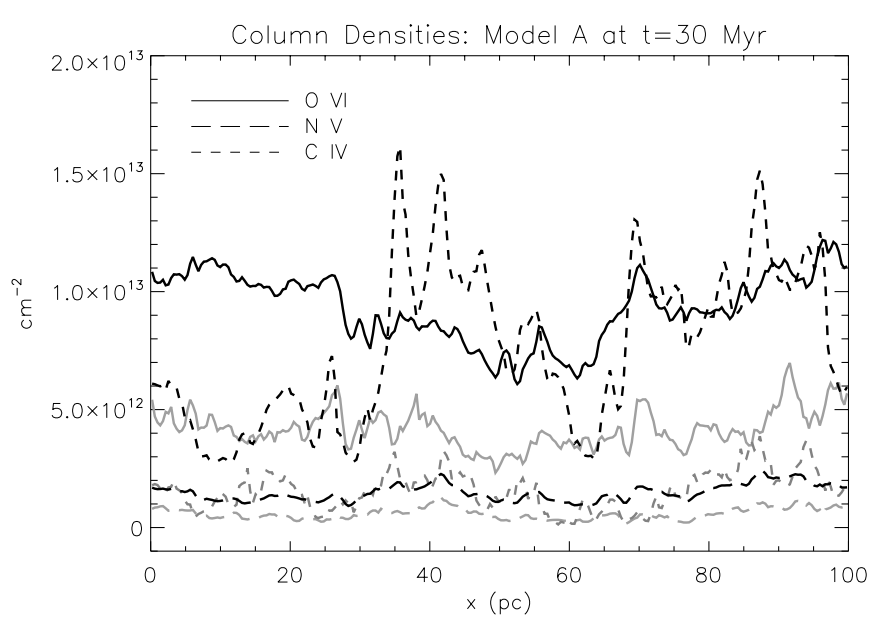

Figure 3. Column densities of C IV (short dashed), Nv (long dashed), and O VI (solid) ions along 256 vertical sightlines through Model A's domain at $t=30$ Myr plotted as a function of position on the $x$-axis. Dark and gray lines are from NEI and CIE calculations, respectively.

the CIE algorithms. This will be examined in more detail in Section 3.1.3. Calculating the column densities from the ion content of the entire domain is too CPU intensive, especially for CIE calculations. Instead, we find them by averaging the column densities of a large number of evenly spaced, infinitely narrow sightlines (256 sightlines for NEI and 86 sightlines for CIE) spread across the domain. For the CIE column densities, we confirm that 86 sightlines are sufficient by comparing their average with that found from 256 sightlines for selected cases. Note that at any given time, the column density found along any given line of sight differs from those found along other lines of sight and thus differs from the average column density. The degree of variation is indicated, for example, in Figure 3, which shows column densities along each of 256 sightlines through the grid at $t=30 \mathrm{Myr}$. Typical variation ranges from as little as standard deviation $/$ mean $=16 \%$ for CIE O vi to as much as $52 \%$ for NEI C IV. The mean, median, standard deviation, and minimum and maximum values of the column density are summarized in Table 2.

\subsubsection{Evolution of Column Densities}

A common question asked while comparing simulations with observations or the results of analytic calculations is "which time frame should be used?" In order to understand the answer, we discuss the time evolution of the high ion column densities here.

The top left panel of Figure 2 shows that the column densities of high-stage ions are approximately 0 at the beginning of the
Table 3

Column Density Increase at Early Times

\begin{tabular}{|c|c|c|c|c|c|}
\hline \multirow[t]{2}{*}{ Model } & \multirow[t]{2}{*}{$\mathrm{NEI} / \mathrm{CIE}$} & \multirow{2}{*}{$\begin{array}{c}\text { Time Period } \\
\text { (Myr) }\end{array}$} & \multicolumn{3}{|c|}{$\begin{array}{l}\text { Column Density Increase } \\
\qquad\left(10^{12} \mathrm{~cm}^{-2}\right)\end{array}$} \\
\hline & & & C IV & $\mathrm{N} \mathrm{V}$ & $\mathrm{OVl}$ \\
\hline A & NEI & {$[0,10]$} & 4.0 & 0.56 & 2.9 \\
\hline A & CIE & {$[0,10]$} & 0.49 & 0.13 & 1.1 \\
\hline B & NEI & {$[0,10]$} & 4.0 & 0.59 & 3.4 \\
\hline B & CIE & {$[0,10]$} & 0.76 & 0.17 & 1.2 \\
\hline $\mathrm{C}$ & NEI & {$[0,2]$} & 0.89 & 0.19 & 0.62 \\
\hline $\mathrm{C}$ & CIE & {$[0,2]$} & 0.17 & 0.041 & 0.38 \\
\hline D & NEI & {$[0,10]$} & 1.1 & 0.18 & 1.1 \\
\hline $\mathrm{E}$ & NEI & {$[0,10]$} & 4.4 & 0.68 & 4.4 \\
\hline $\mathrm{F}$ & NEI & {$[0,10]$} & 1.4 & 0.2 & 1.2 \\
\hline
\end{tabular}

simulation when all of the gas in the domain is either too cool or too hot to be rich in these ions. Once mixing begins, the highstage ion column densities, for both the NEI and CIE variants of Model A increase rapidly. Table 3 presents the amount by which the $\mathrm{CIV}, \mathrm{N} \mathrm{v}$, and $\mathrm{O}$ VI column densities increase between $t=0$ and $t=10 \mathrm{Myr}$. Although the growth rates vary, the column densities of all three ions in both the NEI and CIE variants of Model A continue to grow until $t \approx 20-30 \mathrm{Myr}$, when the depth of the mixed zone peaks for the first time. Between $t \approx 30 \mathrm{Myr}$ and $80 \mathrm{Myr}$, the column densities of all three ions fluctuate. These fluctuations are also seen in the depth of the mixed zone in Figure 1. However, the NEI C IV, in contrast with the NEI $\mathrm{N} v$ and $\mathrm{O}$ VI, climbs to a new plateau at $t \approx 50 \mathrm{Myr}$.

The average C IV column density found using NEI calculations continues to grow until late in the simulation because some of NEI C IV is in the somewhat dense, radiatively cooled region below the interface (see Figure 1) and this region grows, although sporadically, during the simulation time. The gas density in the radiatively cooled region is larger than that in the warmer, actively mixing region below it. Thus, even though the fraction of carbon atoms in the C IV stage (in NEI simulations) is smaller in the cooled gas than in the warm gas (see Figure 1), the number of carbon atoms in the cooled gas is significant. To demonstrate this point, we estimate the average column density of each high-stage ion in the radiatively cooled region and in the actively mixing region at $t=80 \mathrm{Myr}$, when the cooled region is most distinctive. We approximate the cooled region as that between $y=-60$ and $y=0$ pc and the actively mixing region as that between $y=-200$ and $y=-60 \mathrm{pc}$. The column densities are averaged over 256 sightlines for both the NEI and CIE calculations. The average column densities of each species in the cooled and actively mixing region are presented in Table 4. It is shown that in NEI, the average $\mathrm{C}$ IV column density in the cooled region is $47 \%$ as large as that in the mixing region 
Table 4

Volume and Column Densities in Cooled and Mixing Layers of Model A

\begin{tabular}{|c|c|c|c|c|c|c|c|}
\hline \multirow[t]{2}{*}{ Layer } & & \multicolumn{2}{|c|}{ Civ } & \multicolumn{2}{|c|}{$\mathrm{Nv}$} & \multicolumn{2}{|c|}{$\mathrm{O}$ VI } \\
\hline & & NEI & CIE & NEI & CIE & NEI & CIE \\
\hline Cooled $^{\mathrm{a}}$ & $n^{\mathrm{b}}\left(\mathrm{cm}^{-3}\right)$ & $1.94 \times 10^{-8}$ & $2.69 \times 10^{-10}$ & $1.22 \times 10^{-9}$ & $2.50 \times 10^{-11}$ & $4.36 \times 10^{-9}$ & $2.11 \times 10^{-12}$ \\
\hline Layer & $N^{\mathrm{c}}\left(\mathrm{cm}^{-2}\right)$ & $3.60 \times 10^{12}$ & $4.98 \times 10^{10}$ & $2.26 \times 10^{11}$ & $4.63 \times 10^{9}$ & $8.07 \times 10^{11}$ & $3.91 \times 10^{8}$ \\
\hline Mixing $^{d}$ & $n^{\mathrm{b}}\left(\mathrm{cm}^{-3}\right)$ & $1.79 \times 10^{-8}$ & $5.73 \times 10^{-9}$ & $2.80 \times 10^{-9}$ & $9.86 \times 10^{-10}$ & $1.07 \times 10^{-8}$ & $4.76 \times 10^{-9}$ \\
\hline Layer & $N^{\mathrm{c}}\left(\mathrm{cm}^{-2}\right)$ & $7.74 \times 10^{12}$ & $2.48 \times 10^{12}$ & $1.21 \times 10^{12}$ & $4.26 \times 10^{11}$ & $4.62 \times 10^{12}$ & $2.06 \times 10^{12}$ \\
\hline
\end{tabular}

Notes.

a $y \in[-60,0]$ pc.

b Volume density of C IV, N v, and O VI.

${ }^{\mathrm{c}}$ Column density obtained by multiplying volume density with the length of layer, $60 \mathrm{pc}$ for cooled layer and $140 \mathrm{pc}$ for mixing layer.

d $y \in[-200,-60] \mathrm{pc}$.

Table 5

Ratios of Column Densities Calculated Using NEI to Those Calculated Using CIE

\begin{tabular}{|c|c|c|c|c|c|c|c|c|c|c|c|c|}
\hline \multirow[t]{2}{*}{ Model } & \multicolumn{4}{|c|}{ CiV } & \multicolumn{4}{|c|}{$\mathrm{Nv}$} & \multicolumn{4}{|c|}{$\mathrm{O}_{\text {VI }}$} \\
\hline & Mean & Median & $\sigma^{\mathrm{a}}$ & [Min, Max] & Mean & Median & $\sigma^{\mathrm{a}}$ & [Min, Max] & Mean & Median & $\sigma^{\mathrm{a}}$ & [Min, Max] \\
\hline$\overline{A^{b}}$ & 4.64 & 4.62 & 0.44 & {$[3.82,5.67]$} & 2.91 & 2.88 & 0.34 & {$[2.24,3.82]$} & 2.26 & 2.25 & 0.20 & {$[1.83,2.81]$} \\
\hline $\mathrm{B}^{\mathrm{b}}$ & 4.30 & 4.27 & 0.54 & {$[3.30,5.53]$} & 3.00 & 2.89 & 0.42 & {$[2.28,4.03]$} & 2.29 & 2.32 & 0.25 & {$[1.69,2.83]$} \\
\hline $\mathrm{C}^{\mathrm{c}}$ & 3.80 & 3.68 & 0.42 & {$[3.21,4.65]$} & 2.73 & 2.66 & 0.34 & {$[2.27,3.50]$} & 2.09 & 2.03 & 0.28 & {$[1.73,2.68]$} \\
\hline
\end{tabular}

Notes.

${ }^{\text {a }}$ Standard deviation.

b Averaged over $t \in[20,80]$ Myr.

${ }^{c}$ Averaged over $t \in[6,8]$ Myr.

and that the average volume density $\left(\sim 1.94 \times 10^{-8} \mathrm{~cm}^{-3}\right)$ of $\mathrm{C}$ IV ions in the cooled region is even slightly higher than that $\left(\sim 1.79 \times 10^{-8} \mathrm{~cm}^{-3}\right)$ in the mixing region.

The cooled region is poorer in $\mathrm{N} v$ and $\mathrm{O}$ VI than in C IV. Thus, the NEI column densities of $\mathrm{N} v$ and $\mathrm{O} v \mathrm{vI}$ in the cooled region are not as significant as that of C IV. They are $19 \%$ and $17 \%$ of those in the mixing region, respectively. The $\mathrm{CIV}, \mathrm{N} v$, and $\mathrm{O}$ VI in the radiatively cooled zone principally derive from ions that were previously hotter and are now in the process of recombining with free electrons. Since the CIE calculations ignore the plasma's history as they consider only the current temperature, they predict very few highstage ions in the radiatively cooled zone. In CIE, the column densities of all three ions in the cooled region are less than $5 \%$ of those in the mixing region.

\subsubsection{NEI versus CIE}

As soon as mixing begins in our simulations, the NEI calculations predict greater numbers of high-stage ions than the CIE calculations. (Because we assume CIE at the initial time step, the NEI/CIE ratios at $t=0 \mathrm{Myr}$ are unity for all ions.) This is indicated by the second row of panels in Figure 2, and in particular, the leftmost panel in that row, which plots the ratios of column densities predicted by the NEI calculations to the column densities predicted by the CIE calculations for Model A.

Mixing, which proceeds faster than ionization or recombination, forces the gas out of equilibrium. For the first $10 \mathrm{Myr}$, most of the mixed gas is hotter than the CIE temperatures for $\mathrm{C}$ IV and $\mathrm{Nv}$, thus the CIE calculations predict low ion fractions for them. However, larger ion fractions and thus column densities of $\mathrm{CIV}$ and $\mathrm{NV}$ are found in the NEI calculations. These are due to previously cool atoms that have lost a couple of electrons after mixing with hotter gas, but have not yet ionized to their equilibrium levels. Real observations of such gas should find wide absorption and emission features from this hot, "underionized" gas. In addition, the profiles should also be widened by Doppler broadening in the turbulent velocity field.

At their worst, the CIE calculations underpredict the C IV and $\mathrm{N} v$ column densities (relative to the NEI predictions) by factors of $\sim 8$ and $\sim 4$, respectively, but this only occurs during the first $\sim 12 \mathrm{Myr}$ of the simulation. Subsequently, as the mixed zone develops a wider temperature profile, a radiatively cooled zone develops, and more ionization and recombination occurs, the $\mathrm{CIE}$ and NEI predictions begin to track each other with average ratios of 4.6, 2.9, and 2.3 for $\mathrm{C} \mathrm{IV}, \mathrm{NV}$, and $\mathrm{O}$ VI, respectively (see Table 5).

The effects of mixing, radiative cooling, ionization, and recombination can be seen in individual cells. Ideally, we could examine a single cell at various stages in its time evolution, from the moment after it first experienced mixing until long after it radiatively cooled. While this is not practical, it is possible to examine a variety of cells at a single moment in time. Figure 4 shows an expanded image of the domain and points out six cell locations. Cells $1-3$ belong to the lower region of the computational domain, contain newly mixed gas on the boundary of the mixing zone, and are hotter. Most of the material in these cells came from the hot reservoir and minimal radiative cooling has occurred in them. Cells 4 and 5 are deeper in the interior of the mixing zone. Roughly half of their material came from the hot reservoir and half from the cool gas. These cells have radiated away significant fractions of their thermal energy and are now roughly $1 / 2$ the temperature of the hot reservoir. Cell 6 is nearest to the cool gas. Among all of our sample cells, cell 6 has mixed for the largest period of time, entrained the largest fraction of cool gas, and lost the largest fraction of its thermal energy to radiation. It has lost $\geqslant 85 \%$ of its thermal energy to radiation and with a temperature 
Table 6

Cells for Ionization Level Calculations

\begin{tabular}{|c|c|c|c|c|c|c|}
\hline Cell & $\begin{array}{c}\text { Coordinates } \\
(x, y)(\mathrm{pc})\end{array}$ & $\begin{array}{c}n_{H}{ }^{\mathrm{a}} \\
\left(10^{-4} \mathrm{~cm}^{-3}\right)\end{array}$ & $\begin{array}{c}\text { Hot Gas } \\
\text { Mass Fraction }\end{array}$ & $\begin{array}{c}\text { Cool Gas } \\
\text { Mass Fraction }\end{array}$ & $\begin{array}{l}\bar{T}^{\mathrm{b}} \\
(\mathrm{K})\end{array}$ & $\begin{array}{l}\mathrm{T}^{\mathrm{c}} \\
(\mathrm{K})\end{array}$ \\
\hline 1 & $(24.9,-176.0)$ & 0.87 & 0.76 & 0.24 & 760000 & 653000 \\
\hline 2 & $(34.3,-181.4)$ & 1.72 & 0.65 & 0.35 & 650000 & 462000 \\
\hline 3 & $(40.7,-148.6)$ & 1.83 & 0.60 & 0.40 & 600000 & 371000 \\
\hline 4 & $(59.5,-71.8)$ & 2.51 & 0.44 & 0.56 & 441000 & 251000 \\
\hline 5 & $(78.0,-59.5)$ & 3.65 & 0.36 & 0.64 & 361000 & 152000 \\
\hline 6 & $(61.9,-40.9)$ & 40.9 & 0.11 & 0.89 & 110890 & 15000 \\
\hline
\end{tabular}

Notes.

${ }^{a}$ Hydrogen number density.

${ }^{\mathrm{b}}$ Mixed temperature if there had been no radiative cooling. It is calculated from $\bar{T}=T_{\text {hot }} \times f_{\text {hot }}+T_{\text {cool }} \times f_{\text {cool }}$ where $T_{\text {hot }}=1.0 \times 10^{6} \mathrm{~K}$, $T_{\text {cool }}=1.0 \times 10^{3} \mathrm{~K}$, and $f_{\text {hot }}$ and $f_{\text {cool }}$ are mass fractions of hot and cool gas, respectively.

c Temperature measured at the cell from the simulation.

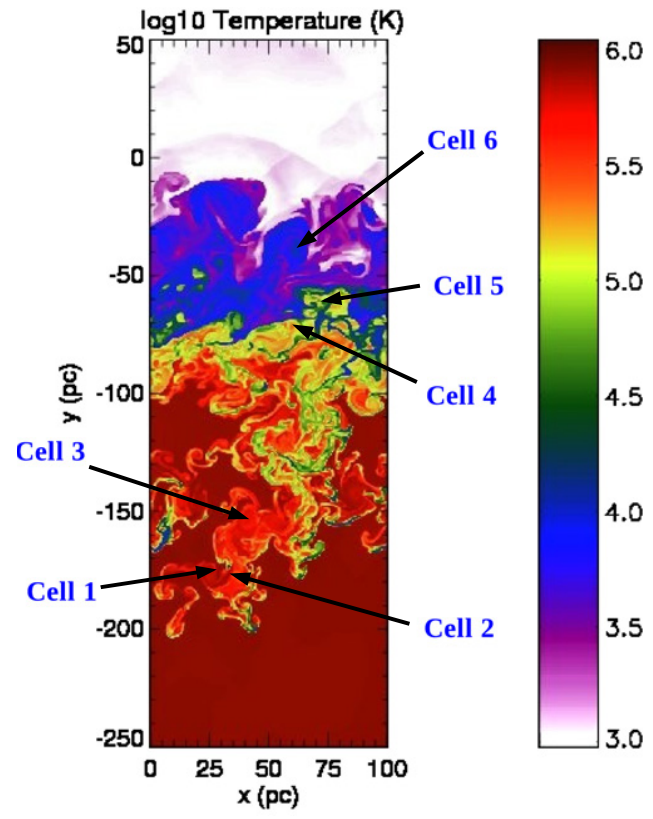

Figure 4. Cell locations for ionization level calculations for carbon, nitrogen, and oxygen. In this temperature map for Model A at $t=80 \mathrm{Myr}$, six cells are identified. The information for each cell is given in Table 6 and the ionization level calculations are shown in Figure 5.

(A color version of this figure is available in the online journal.)

of $\sim 15,000 \mathrm{~K}$, exemplifies the radiatively cooled zone at the "base" of the mixed zone.

Table 6 lists the locations (Column 2) and hydrogen number densities (Column 3) of these cells. The FLASH code allows us to trace the mass fraction of the initial cool and hot gas at each cell over time. The mass fraction of cell material that originally came from the hot reservoir $\left(f_{\text {hot }}\right.$, Column 4$)$ and the mass fraction from the cool reservoir $\left(f_{\text {cool }}\right.$, Column 5$)$ are also listed in Table 6. From these mass fractions, we calculate the temperature that the gas would have had if radiative cooling had not occurred, $\bar{T}=T_{\text {hot }} \times f_{\text {hot }}+T_{\text {cool }} \times f_{\text {cool }}$, where $T_{\text {hot }}=1.0 \times 10^{6} \mathrm{~K}$ and $T_{\text {cool }}=1.0 \times 10^{3} \mathrm{~K}$. This temperature is tabulated in (Column 6) in Table 6. The measured temperature at each cell from the simulation ( $T$, Column 7 ) is the temperature that the mixed gas reaches after the radiative cooling is in effect. Comparing $\bar{T}$ (Column 6) and $T$ (Column 7) shows how significantly radiative cooling has lowered the temperature of the mixed gas.
The hot reservoir is in or near CIE and thus mixing such gas into a cell raises the fraction of very highly ionized atoms in the cell. For this reason, cell 1 contains large numbers of hydrogenlike, helium-like, and fully stripped carbon, as indicated by the top left panel of Figure 5. (Figure 5 shows the ion fractions, calculated from both the NEI and CIE algorithms, except for the NEI ion fractions of N VIII, O VIII, and OIX, which are not included in the plot although they were traced in our NEI calculations.) The greater prevalence of very high ions in the NEI case than in the CIE case is a sign of delayed recombination in NEI calculations; the recombination rate is slower than the mixing rate.

As the temperature drops (progressing from cell 1 to larger numbered cells) due to radiative cooling and advection of cooler gas, the fraction of very high ions predicted by CIE calculations decreases much faster than those predicted by NEI calculations. Because the NEI recombination rate lags the cooling rate so severely, we can find C IV in the $15,000 \mathrm{~K}$ gas in cell 6, next to the cool gas. This is consistent with the sudden increase of column density of C IV at later times shown in the top left panel of Figure 2 (Section 3.1.2).

Similarly, when cells advect cool gas, they gain low ions, which raise their low ion fractions. These ions are slow to ionize, causing the fractions of NEI CIV, Nv, and $\mathrm{O}$ VI in cell 1 to be significantly greater than those predicted by CIE calculations. In cells 4 and 5, the NEI predictions for once, twice, and thrice ionized carbon, nitrogen, and oxygen are more similar to those of CIE calculations, but this is not solely due to recombinations. Instead, it occurs because radiative cooling has lowered the gas temperature enough for the CIE fractions of low ions to converge with the NEI ion fractions. Such gas is not actually in ionization equilibrium, as is apparent from the fact that the fractions of high ions greatly exceed those predicted by CIE calculations.

The ionization behavior in our NEI simulations is consistent with previous studies with NEI calculations. Ballet et al. (1986) studied the evaporation of a spherical gas cloud and found that in NEI calculations, ionization to He-like stages is delayed. In their calculations, the gas is heated via conduction, while in our simulations, the gas is heated due to mixing. Boehringer \& Hartquist (1987) added radiative cooling to the calculations of Ballet et al. (1986) and found a similar delayed ionization trend in the conductive interface of the evaporating cloud. Radiative cooling plays a more important role in the production of high ions when hot $\left(\geqslant\right.$ a few times $\left.10^{6} \mathrm{~K}\right)$ gas cools radiatively. Recently, Gnat \& Sternberg (2007) numerically calculated the ionization states and corresponding radiative cooling rates 

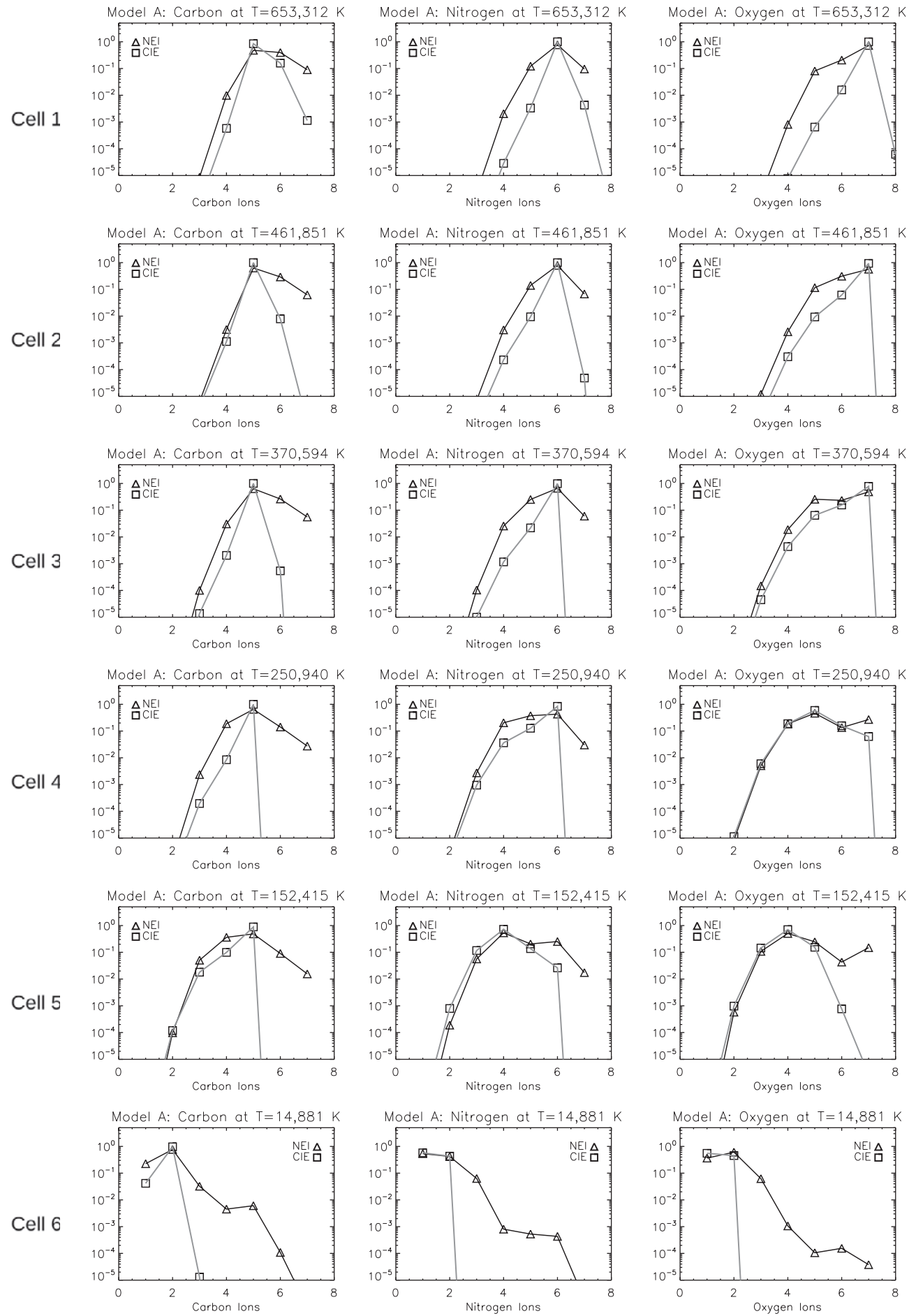

Figure 5. Model A at $t=80 \mathrm{Myr}$ : fraction of atoms in various ionization levels vs. ionization level for three elements, carbon, nitrogen, and oxygen. The ionization levels are indicated by $1,2,3$, etc., on the abscissa, where, for example, 1 on a carbon plot refers to $\mathrm{C}$. Values from NEI calculations are indicated by triangles, while values from CIE calculations are indicated by squares. The NEI ion fractions of N VIII, O VIII, and O IX are not included in the plot although they were traced in our NEI calculations. Note that the CIE ion fractions at low temperatures are as small as the numerical precision in our CIE calculation code. The top row of panels pertains to cell 1 in Figure 4, while the subsequent panels pertain to cells 2-6 in Figure 4. Information about each zone is given in Table 6.

for the elements $\mathrm{H}, \mathrm{He}, \mathrm{C}, \mathrm{N}, \mathrm{O}, \mathrm{Ne}, \mathrm{Mg}, \mathrm{Si}, \mathrm{S}$, and $\mathrm{Fe}$. They included recent atomic data and investigated the effect of various metallicities. Their NEI calculations confirm that recombination is delayed relative to the radiative cooling rate. Similar recombination lags have been shown in earlier NEI calculations, which are summarized in Gnat \& Sternberg (2007). Previous CIE calculations in the context of radiatively cooled gas are also reviewed in Gnat \& Sternberg (2007).

\subsubsection{Ratios between Column Densities of Different Ions}

The third left to fifth left panels in Figure 2 show ratios between the column densities of different ions, $N(\mathrm{C}$ IV $) / N(\mathrm{~N} \mathrm{v})$, $N(\mathrm{O} v \mathrm{II}) / N(\mathrm{C} \mathrm{IV})$, and $N(\mathrm{O} \mathrm{vI}) / N(\mathrm{~N} \mathrm{v})$, respectively, calculated both from NEI and CIE for Model A. The ratios follow a similar trend as the column density evolution (top left panel) and NEI/ CIE ratios for each ion (second left panel) such that they start to 
Table 7

Column Density Ratios between Different Ions

\begin{tabular}{|c|c|c|c|c|c|c|c|c|c|c|c|c|}
\hline \multirow{2}{*}{$\begin{array}{c}\text { Model } \\
\text { NEI/CIE }\end{array}$} & \multicolumn{4}{|c|}{$N(\mathrm{C}$ IV $) / N(\mathrm{~N} \mathrm{v})$} & \multicolumn{4}{|c|}{$N(\mathrm{O}$ vI $) / N(\mathrm{C}$ IV $)$} & \multicolumn{4}{|c|}{$N(\mathrm{O}$ vI $) / N(\mathrm{~N} \mathrm{v})$} \\
\hline & Mean & Median & $\sigma^{\mathrm{a}}$ & [Min, Max] & Mean & Median & $\sigma^{\mathrm{a}}$ & [Min, Max] & Mean & Median & $\sigma^{\mathrm{a}}$ & [Min, Max] \\
\hline $\mathrm{A}^{\mathrm{b}} \mathrm{NEI}$ & 6.72 & 6.75 & 0.84 & {$[5.04,8.61]$} & 0.70 & 0.66 & 0.21 & {$[0.42,1.22]$} & 4.61 & 4.38 & 0.96 & {$[3.14,7.23]$} \\
\hline CIE & 4.27 & 4.07 & 0.94 & {$[2.39,7.10]$} & 1.46 & 1.38 & 0.48 & {$[0.65,2.96]$} & 5.88 & 5.72 & 1.00 & {$[4.26,8.57]$} \\
\hline $\mathrm{B}^{\mathrm{b}} \mathrm{NEI}$ & 6.77 & 7.05 & 1.10 & {$[4.20,8.25]$} & 0.68 & 0.66 & 0.21 & {$[0.41,1.23]$} & 4.41 & 4.30 & 0.84 & {$[3.18,6.76]$} \\
\hline CIE & 4.76 & 4.73 & 1.01 & {$[3.01,6.92]$} & 1.28 & 1.28 & 0.43 & {$[0.63,2.32]$} & 5.77 & 5.71 & 1.15 & {$[3.68,8.69]$} \\
\hline $\mathrm{C}^{\mathrm{c}} \mathrm{NEI}$ & 6.42 & 6.43 & 0.34 & {$[5.90,6.98]$} & 0.59 & 0.55 & 0.08 & {$[0.51,0.72]$} & 3.76 & 3.69 & 0.47 & {$[3.09,4.56]$} \\
\hline CIE & 4.68 & 4.57 & 0.92 & {$[3.49,6.32]$} & 1.08 & 1.13 & 0.19 & {$[0.71,1.31]$} & 5.04 & 5.10 & 1.19 & {$[3.25,7.13]$} \\
\hline $\mathrm{D}^{\mathrm{d}} \mathrm{NEI}$ & 8.17 & 8.33 & 0.94 & {$[6.08,10.00]$} & 0.61 & 0.49 & 0.18 & {$[0.42,0.94]$} & 4.81 & 4.36 & 1.03 & {$[3.63,6.85]$} \\
\hline $\mathrm{E}^{\mathrm{b}} \mathrm{NEI}$ & 7.02 & 7.17 & 0.96 & {$[4.86,8.69]$} & 0.67 & 0.65 & 0.20 & {$[0.38,1.25]$} & 4.56 & 4.33 & 1.02 & {$[3.17,7.08]$} \\
\hline $\mathrm{F}^{\mathrm{b}} \mathrm{NEI}$ & 10.25 & 10.37 & 1.06 & {$[7.16,12.55]$} & 0.55 & 0.54 & 0.11 & {$[0.40,0.96]$} & 5.61 & 5.56 & 0.84 & {$[4.39,8.55]$} \\
\hline
\end{tabular}

Notes.

a Standard deviation.

b Averaged over $t \in[20,80]$ Myr.

c Averaged over $t \in[6,8]$ Myr.

d Averaged over $t \in[30,80] \mathrm{Myr}$.

stabilize around $t=20$ Myr. Estimated mean, median, standard deviation, and minimum and maximum values of the column density ratios during the time period of $t=20$ to $t=80 \mathrm{Myr}$ for Model A are given in Table 7.

The ratios of various ions' column densities are the most frequently used diagnostics for evaluating observations because models that are based upon differing physical processes predict different values. In the following sections, we will estimate the column density ratios between different ions for all of our model simulations and summarize them in Table 7. Comparisons between our results and other models, including the turbulent mixing calculations of Esquivel et al. (2006) and Slavin et al. (1993) will be given in Section 4.

\subsection{Model B: Higher Resolution than Model A}

In Model B, the smallest cells are half the height and half the width of the smallest cells in Model A. All of the other parameters are the same as in Model A. The high ion column densities and ratios predicted for the Model B simulation are shown in the middle column of Figure 2. The panels are placed in the same order as used for Model A (left column). A comparison between the left and middle column shows that the results of our high-resolution simulation (Model B) are very similar to those of the reference simulation (Model A). The top left and top middle panels show that the evolution of column densities in Model B follows the same trends as Model A such that all column densities, both in NEI and CIE, continue to increase until $t \approx 20-30 \mathrm{Myr}$ and then fluctuate between $t=20$ and $t=80 \mathrm{Myr}$. Similarly, the column density ratios between NEI and CIE (second left and second middle panels) stabilize after $t=20 \mathrm{Myr}$ for both models. As in Model A, the NEI calculations predict more high ions than do the CIE calculations. Between $t=20$ and $t=80 \mathrm{Myr}$, the mean/ standard deviations of the ratios of column densities calculated using NEI to the column densities calculated using CIE are $4.30 / 0.54,3.00 / 0.42$, and $2.29 / 0.25$ for $\mathrm{C}$ IV, $\mathrm{Nv}$, and $\mathrm{O}$ VI, respectively (Table 5). These values in Model B are close to those calculated in Model A (Section 3.1.2). From the information in the third middle to fifth middle panels, we tabulate the mean, median, standard deviation, and minimum and maximum values of $N(\mathrm{C}$ IV $) / N(\mathrm{~N} \mathrm{v}), N(\mathrm{O} \mathrm{vI}) / N(\mathrm{C} \mathrm{IV})$, and $N(\mathrm{O} \mathrm{vI}) / N(\mathrm{~N} \mathrm{v})$, both for the NEI and CIE predictions. The values are given in
Table 7 and confirm the similarity between Model B and Model A.

Even though the overall results of Model B are very similar to those of Model A, we do find that resolution affects the temperature of the mixed gas and therefore the column densities at early times. Between $t=0$ and $t=10 \mathrm{Myr}$, the column densities of each ion in Model B (top middle panel in Figure 2) increase by $4.0 \times 10^{12}\left(7.6 \times 10^{11}\right), 5.9 \times 10^{11}\left(1.7 \times 10^{11}\right)$, and $3.4 \times 10^{12}\left(1.2 \times 10^{12}\right) \mathrm{cm}^{-2}$ for $\mathrm{C} \mathrm{IV}, \mathrm{N} \mathrm{v}$, and $\mathrm{O}$ VI, respectively, in NEI (CIE) (see Table 3). Although these values for Model B are close to those from Model A (Section 3.1.2) in both NEI and CIE, the increase in the column densities in Model B is slightly larger than that in Model A for all ions, which indicates that hot and cool gas mix slightly faster in the high-resolution simulation between $t=0$ and $t=10 \mathrm{Myr}$. Because C IV in CIE traces the mixed gas around $10^{5} \mathrm{~K}$, the gas around this temperature is most affected. It is produced much faster in the high-resolution simulation.

\subsection{Model C: 1/10 Scale Simulation}

In Model C, we run the simulation for $1 / 10$ th the time period $(8 \mathrm{Myr})$ in a $1 / 10$ th scale computational domain $(10 \times 30 \mathrm{pc})$ than in Model A. The purpose of performing this model is to check whether the results of Model A (our reference simulation) are still valid on a smaller scale and to study whether the difference between NEI and CIE begins at early times in the smaller domain. This is an important issue because we include radiative cooling which is not scale invariant. If radiative cooling were not included, the smaller spatial scale simulation would be identical to the larger scale simulation because the spatial coordinates and time are proportional to each other in the hydrodynamics equations without any dissipative terms, making the calculations scale invariant. For those researchers who are interested in the early evolution of the mixing zone, Model C can be seen as a close-up, with greater spatial resolution and smaller time periods.

The column densities and ratios predicted for Model $\mathrm{C}$ are shown in the right column of Figure 2, in which the plots are placed in the same order (from top to bottom) as for Models A and B (left and middle columns in Figure 2). At early times, the column densities of each ion in Model C (top right panel) evolve in a similar manner as those in Models A and B, such that they 

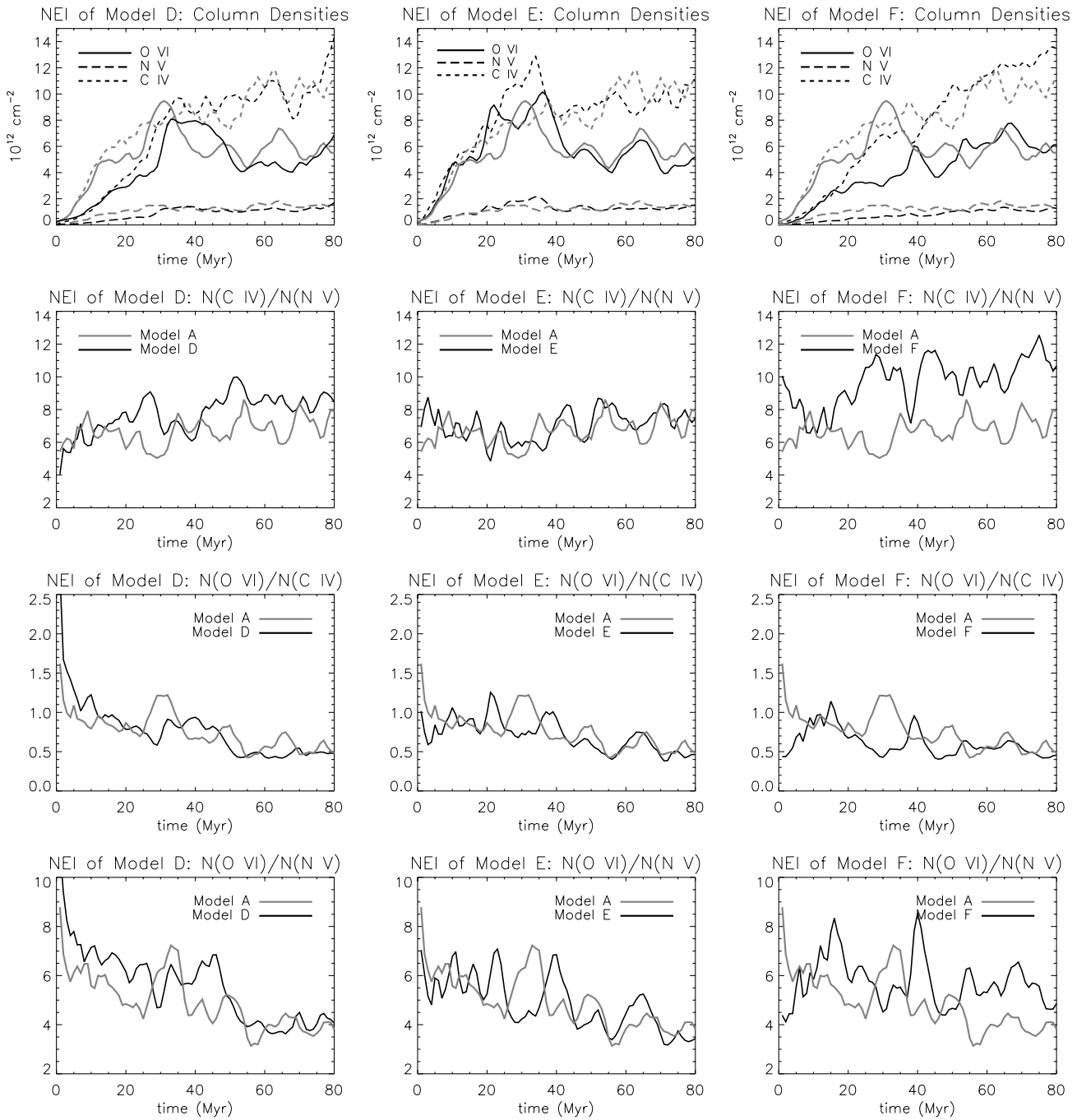

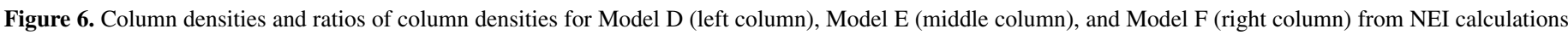

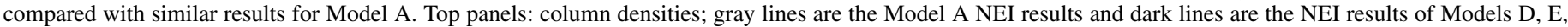
and $\mathrm{F}$ in the left, middle, and right panel, respectively. Second, third, and fourth panels: column density ratios of $\mathrm{C}$ IV/N v, O vi/C IV, and O vi/N v, respectively.

continue to increase until $t \approx 3 \mathrm{Myr}$, i.e., $\sim 1 / 10$ of the onset periods in Models A and B. The column density rises for each ion both in NEI and CIE for Model C are given in Table 3. These increases are close to those in Models A and B over $10 \mathrm{Myr}$, showing that the early evolution of the mixing layer does not depend on the scale as long as the radiative cooling rate is not faster than the mixing rate.

There are ways in which Model $\mathrm{C}$ behaves more like Model A during its first $8 \mathrm{Myr}$ than like a timescaled version of Model A. In Model A, the depth of the mixed zone and the column densities of Li-like ions increase steadily for the first $8 \mathrm{Myr}$ of the simulation. Similarly, they increase in Model C during this time period. But, if Models $\mathrm{A}$ and $\mathrm{C}$ acted like timescaled version of each other, then the depth of the mixed zone and the column densities of Li-like ions would stall at $2 \mathrm{Myr}(=20 / 80 \times 8 \mathrm{Myr})$ in Model C, which they do not. Model C's mixing zone grows so steadily that it overflows the computational domain by 8 Myr. Model C, therefore, does not run long enough to develop a C IV-rich, radiatively cooled $\left(T<2 \times 10^{4} \mathrm{~K}\right.$ ) layer similar to that which appears in Model A around 10 Myr. As a result, most of the existing $\mathrm{C}$ IV in Model $\mathrm{C}$ resides in the mixed layer of actively cooling gas $\left(T \geqslant 10^{5} \mathrm{~K}\right)$, the same region where $\mathrm{O}$ VI resides. The same can be said of Model A at early times.

\subsection{Models D, E, and F: Modified Initial Configurations}

Models D, E, and F are presented in order to address the following questions regarding the physical conditions of the mixing layer. Do different physical conditions affect the growth of mixing layers and do different physical conditions affect the column density ratios such that the observed ratios can be used as diagnostics of mixing layers? Answering these questions, we find that modifying the initial amplitude of the disturbance between the hot and cool gases does not change the characteristics of mixing layer (see Section 3.4.2 regarding Model E). However, adjusting the speed difference between the hot and cool layers (see Section 3.4.1 regarding Model D) and the temperature of the hot gas (see Section 3.4.3 regarding Model F) affect the column density ratios. Because NEI provides more realistic diagnostics than CIE, only the NEI results of these models are compared with those of Model A, the reference simulation. Figure 6 shows the results of Models D, E, and F in the left, middle, and right columns, respectively, together with the NEI results of Model A for comparison.

\subsubsection{Model D: Slow Speed}

In Model D, the hot and cool gases slide past each other with a smaller speed $\left(50 \mathrm{~km} \mathrm{~s}^{-1}\right)$ than in Model A $\left(100 \mathrm{~km} \mathrm{~s}^{-1}\right)$. 
Mixing occurs more slowly in Model D than in Model A because mixing is caused by shear instabilities, which grow with the speed difference between the two bodies. As a result, the earlytime high ion column densities build up more slowly (see the top left panel of Figure 6 and Table 3). Although slightly delayed, the column densities of Li-like ions in Model D do approach those of Model A by the middle of the simulation time.

The column densities from Model D continue to increase until $t \approx 30 \mathrm{Myr}$ when they begin to fluctuate. We estimate the column density ratios between different ions for Model D between $t=30$ and $t=80 \mathrm{Myr}$ and list them in Table 7 . Compared with Model A during a similar time frame, Model D has a higher average $N(\mathrm{C}$ Iv $) / N(\mathrm{~N} v)$ ratio but a lower average $N(\mathrm{O}$ vI $) / N(\mathrm{C}$ IV $)$ ratio, while the $N(\mathrm{OVI}) / N(\mathrm{Nv})$ ratios are similar. The noticeable variations in the column density ratios due to only $50 \mathrm{~km} \mathrm{~s}^{-1}$ of velocity difference suggest that the ratios may serve as diagnostics of velocity when the velocity difference between the hot and cool gas is large (see Section 3.4.4).

\subsubsection{Model E: Large Initial Amplitude}

The sine wave shape of the interface between the hot and cool gases is larger in Model E than in Model A, allowing us to test the effect of the interface's curvature. We find that Model E's characteristics, including depth of mixing region, high ion column densities, and column density ratios, are similar to those of Model A (see Figure 6 and Table 3). These results show that the perturbation amplitudes in the initial interface between the hot and cool gas do not significantly affect the physical properties of the turbulent mixing layer as long as mixing is efficient.

\subsubsection{Model F: Hot Gas with Higher Temperature}

In Model F, the hot gas has a higher temperature $\left(3 \times 10^{6} \mathrm{~K}\right)$ than in Model A. Because we require the thermal pressure of the initial hot layer to equal that of the cool gas layer, the hot gas in Model F has a smaller density $\left(\frac{1}{3} \times 10^{-4} \mathrm{H}_{\text {atoms cm}}{ }^{-3}\right)$ than that in Model A.

The turbulent mixing layer in Model $\mathrm{F}$ evolves differently than that in Model A; the high ion column densities increase at a slower pace and continue to rise throughout the simulation period (see Table 3 and Figure 6). Furthermore, the newly mixed gas is hotter and the radiatively cooled gas $\left(T \leqslant 2 \times 10^{4} \mathrm{~K}\right)$ is shallower in Model F than in Model A because it takes longer for the hotter mixed gas in Model F to cool. We find that the high ion population is low in the hotter, newly mixed gas in Model $\mathrm{F}$ than in Model A. This effect seems to yield larger $N(\mathrm{C}$ IV $) / N(\mathrm{~N} \mathrm{v}$ ) and $N(\mathrm{O}$ vi $) / N(\mathrm{~N}$ v) ratios than in Model A (see Figure 6 and Table 7). Model F's $N(\mathrm{C} \mathrm{Iv}) / N(\mathrm{~N} v)$ ratio is greater than that of Model A for nearly the entire simulation. Thus, this ratio, especially if used in conjunction with the $N(\mathrm{O} v \mathrm{vI}) / N(\mathrm{~N} \mathrm{v})$ ratio may serve as a diagnostic of the hot gas temperature when interpreting observations of mixing gas.

\subsubsection{Diagnostics for Observations}

The column densities on sightlines that run perpendicular to the turbulent mixing layers are on the order of $10^{13} \mathrm{~cm}^{-2}$ for $\mathrm{C}$ IV, $10^{12} \mathrm{~cm}^{-2}$ for $\mathrm{Nv}$, and $5 \times 10^{12} \mathrm{~cm}^{-2}$ for $\mathrm{O} \mathrm{VI}$ in our model simulations (except Model C). In real observations, the sightlines probably pass through the layers at sharper angles and intersect multiple mixing layers or multiple portions of curved mixing layers that surround individual clouds. Each of these effects increases the column densities by a multiplicative factor. When comparing with an observation, the model column density ratios are more useful than the model column densities because the multiplicative factor is unknown. In Section 4.2, we compare the ion ratios from our simulations with actual observations. Here, we discuss the use of column density ratios as diagnostics of the physical conditions producing the turbulent mixing layers.

The results of Models D and F reveal that the ratios between the column densities of different ions can be used as diagnostics for the shear speed and hot gas temperature. If the $N(\mathrm{C}$ IV $) /$ $N(\mathrm{~N} \mathrm{v})$ and $N(\mathrm{C}$ IV $) / N(\mathrm{O}$ VI $)$ ratios along a sightline through a specific cloud are larger than those along sightlines through other clouds while the $N(\mathrm{O} v \mathrm{v}) / N(\mathrm{Nv})$ ratio is similar along all of these sightlines, then it is likely that the mixing layer on the first cloud forms with a slower sheer speed (Model D). Another possible diagnostic comes from Model F. If observations along one sightline have higher $N(\mathrm{C}$ Iv $) / N(\mathrm{~N} \mathrm{v})$ and $N(\mathrm{O}$ vI $) / N(\mathrm{~N} \mathrm{v})$ ratios but lower $N(\mathrm{O} \mathrm{VI}) / N(\mathrm{C}$ IV $)$ ratios compared with observations of other clouds, it is possible to assume that the first mixing layer formed in hotter ambient gas than the others.

Generally, more C IV is produced in the NEI calculations of the models than in the CIE calculations, especially in the radiatively cooled regions. This high population of $\mathrm{C}$ IV affects the column density ratios between different ions. However, C IV can also be produced by photoionization and is produced more easily than other high ions if there are nearby photoionizing sources because the photoionization threshold of C IV is only $48 \mathrm{eV}$. Therefore, care must be taken when evaluating observational results.

\section{DISCUSSION}

The column densities of high ions located in the Galactic halo are observable from the absorption lines in the spectra of halo stars and extragalactic objects. Here, we compare observed high ion column densities and their ratios with those estimated from our model simulations. We also compare our results with predictions from other turbulent mixing models and from other sorts of models for high-stage ions. However, there are some uncertainties in estimating the column densities from model calculations. Therefore, we discuss these uncertainties before addressing how much they affect the results of model calculations and the comparisons with observations.

\subsection{Uncertainties in Model Calculations}

High ion column density predictions are strongly dependent on the assumed metallicity of the gas experiencing the modeled physical processes. However, the metallicity in the Galactic halo is not well constrained and the uncertainty increases from the Milky Way to the intergalactic medium and external galaxies. Therefore, the column density calculations vary according to the metallicity used in the model.

We find that most previous model calculations used solar metallicity, which is sometimes better constrained by meteorite measurements than other methods, although the abundances of carbon, nitrogen, and oxygen still have large uncertainties, even in this method. Because metallicity measurements have been revised repeatedly over the years, different model calculations used slightly different metallicities, especially for carbon, nitrogen, and oxygen. For example, the turbulent mixing calculations of Slavin et al. (1993) used the solar photospheric metallicities of Grevesse (1984) and assumed that the cool gas has depleted 
Table 8

Abundances Normalized to Allen (1973)

\begin{tabular}{lllllll}
\hline \hline Reference & $\mathrm{C}^{\mathrm{a}}$ & $\mathrm{N}^{\mathrm{a}}$ & $\mathrm{O}^{\mathrm{a}}$ & $\mathrm{C} / \mathrm{N}$ & $\mathrm{O} / \mathrm{C}$ & $\mathrm{O} / \mathrm{N}$ \\
\hline 1 & 1.34 & 1.00 & 1.12 & 1.34 & 0.83 & 1.12 \\
2 & 1.48 & 1.07 & 1.23 & 1.38 & 0.83 & 1.15 \\
3 & 1.10 & 1.23 & 1.29 & 0.89 & 1.17 & 1.05 \\
4 & 1.07 & 1.02 & 1.12 & 1.05 & 1.05 & 1.10 \\
5 & 0.74 & 0.93 & 0.74 & 0.80 & 1.00 & 0.80 \\
6 & 0.054 & 0.013 & 0.12 & 4.15 & 2.22 & 9.23 \\
7 & 0.74 & 0.66 & 0.69 & 1.12 & 0.93 & 1.05 \\
\hline
\end{tabular}

Notes.

a Carbon, nitrogen, and oxygen abundance ratio to that in Allen (1973) for each reference. In Allen (1973), carbon, nitrogen, and oxygen abundance is $3.31 \times 10^{-4}, 9.12 \times 10^{-5}$, and $6.61 \times 10^{-4}$ per hydrogen atom, respectively.

References. (1) Anders \& Ebihara (1982) metallicities used in Boehringer \& Hartquist (1987) and in Slavin \& Cox (1993); (2) Grevesse (1984) used in Borkowski et al. (1990) and Slavin et al. (1993); (3) Anders \& Grevesse (1989) used in Sutherland \& Dopita (1993), Shelton (1998), and Esquivel et al. (2006); (4) Grevesse et al. (1996) used in Indebetouw \& Shull (2004a); (5) Fox et al. (2004): adopted solar metallicity from Holweger (2001), Allende Prieto et al. (2002), and Allende Prieto et al. (2001); (6) Fox et al. (2004): Complex C metallicity estimated along the PG 1259+593 sightline; (7) Asplund et al. (2005): solar photospheric metallicity.

metallicities (such that $50 \%$ of carbon, nitrogen, and oxygen are depleted into dust grains) while all dust grains are destroyed in the initial hot gas. In contrast, the CIE calculations (Benjamin et al. 2001) in the numerical study of turbulent mixing layers of Esquivel et al. (2006) used the updated solar photospheric metallicity data from Anders \& Grevesse (1989) which have different abundances for carbon, nitrogen, and oxygen from Grevesse (1984) (see Table 8 for the difference between these two metallicities).

For consistency between our NEI and CIE calculations, we use the cosmic abundances of Allen (1973) because they are the default abundances both in the FLASH NEI module and in the HEASARC version of the Raymond and Smith code, which we use for our CIE calculations. Note that the abundances of carbon, nitrogen, and oxygen from most of the references (before the year 2000) are higher than those from Allen (1973) although recent measurements (Asplund et al. 2005; Allende Prieto et al. 2002, 2001; Holweger 2001) measured lower abundances of these elements. Table 8 shows the ratios of solar metallicities from various references to the abundance from Allen (1973) for carbon, nitrogen, and oxygen. The ratios for fractions between different atoms such as carbon/nitrogen, oxygen/carbon, and oxygen/nitrogen are also presented. They can be used to convert our column density ratios to those for different metallicities. For example, if the solar metallicities from Anders \& Grevesse (1989) were desired instead of those from Allen (1973), then our column density ratio predictions would need to be multiplied by $0.89,1.17$, and 1.05 for $N(\mathrm{C}$ IV $) / N(\mathrm{~N} \mathrm{v}), N(\mathrm{O}$ vi $) / N(\mathrm{C}$ IV $)$, and $N(\mathrm{O}$ vI $) / N(\mathrm{~N} \mathrm{v})$, respectively. Note that this conversion for different abundances is approximate for the complete effects of abundances on the high ions (and their ratios) because abundances of metal ions also affect the cooling rates thus influencing the dynamical evolution of the gas containing the high ions. In optically thin gas, large abundances of metal ions increase the cooling rates because the dominant cooling process is resonant line emission from these metal ions. As mentioned in Section 2, more complete future studies are required to address the full effects of abundances of all relevant ions on the cooling rates.
Photoionization also significantly affects the column densities of high ions and the column density ratios between different ions although we do not consider its effect in our simulations. Because the photoionization energy of C IV is much lower than those of $\mathrm{N} \mathrm{V}$ and $\mathrm{O}$ VI, it is likely that photoionization preferentially enhances the $\mathrm{C}$ IV column densities. In this case, as already mentioned in Section 3.4.4, it is not easy to distinguish observationally between collisionally ionized C IV in the radiatively cooled part of the mixing layer and photoionized C IV. Note that Slavin et al. (1993) considered the effect of photoionization for the case where photons radiated from the mixed layer irradiate the cool gas.

Besides the above two, there are two more uncertainties in dynamical models of turbulent mixing layers: time and sightline dependence. Column densities can be calculated along specific sightlines at specific times and can be directly compared with the observed ones. However, the computed column densities vary significantly with viewing angle and position, and in dynamical models also vary with time. For example, Figure 3 and Table 2 show column density variations between sightlines at a specific time $(t=30 \mathrm{Myr})$, while the top panels in Figures 2 and 6 show variations of sightline averaged column densities over time. In general, when these sources of variation are taken into account, the computed column densities from the dynamical models have wider ranges of predicted values than those from the static models.

It is necessary to address again the issue of our simulated turbulent mixing layers reaching steady states, particularly the question of whether the evolution of column densities and their ratios can be applied to interstellar and intergalactic clouds that are relatively small so travel the length of the cloud in less than 80 Myr (see Section 3.1). In our model simulations, the column densities and their ratios evolve until $80 \mathrm{Myr}$ (except in Model C) which corresponds to a length of roughly $8 \mathrm{kpc}$ for the $100 \mathrm{pc}$ long mixing layers and a speed of $100 \mathrm{~km} \mathrm{~s}^{-1}$. This size may be too large for the realistic size of the clouds in the Milky Way although it is a possible size for some of HVCs relatively far away in the halo such as Complex $\mathrm{C}$ whose distance and area are measured as roughly $10 \mathrm{kpc}$ and $3 \times 15 \mathrm{kpc}^{2}$, respectively (Thom et al. 2008). In the case that the size of the cloud in the Milky Way is much smaller than $8 \mathrm{kpc}$, care needs to be taken when using the column densities of high ions in our simulations because the column densities continue to increase during the early times of the simulation. However, the ratios between high ion column densities settle down quickly and do not vary significantly at later times implying that the ratios in Table 7 are applicable at early times as well and for small clouds. In addition, the Model C simulations which use a smaller domain and concentrate on the earlier times show that the ratios between high ion column densities settle down very quickly (around $t=2 \mathrm{Myr}$, which is similar to the cooling timescale in Section 3.1) and remain similar afterward, supporting the use of the values in Table 7 for earlier times (and for smaller clouds).

Finally, we discuss the effects of some physical processes that we do not include in our simulations but could affect the results of our simulations. First, the results of our two-dimensional simulations for the NEI calculations of high ion column densities could be different from more realistic three-dimensional simulations because it has been known that turbulent motion due to instabilities in three dimensions is different from that in two dimensions (Hussain 1984; Bayly 1986; Craik \& Criminale 1986). The magnetic field is known to suppress turbulence both in two dimensions and three dimensions (Frank et al. 1996; Jones et al. 


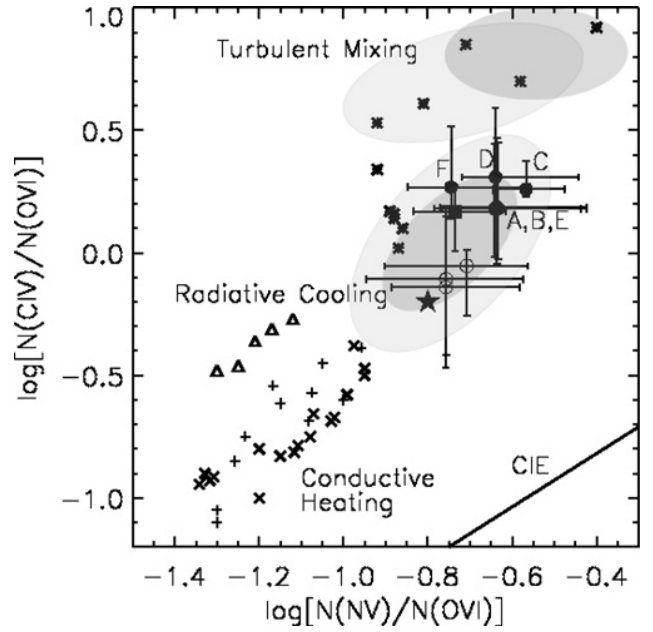

Figure 7. Column density ratios between different ions in $\log [N(\mathrm{Nv}) /$ $N(\mathrm{O}$ VI $)]-\log [N(\mathrm{C}$ IV $) / N(\mathrm{O}$ VI $)]$ space. Re-plotted from Figure 1 of Indebetouw $\&$ Shull (2004a), with additions. Models: radiative cooling of Galactic fountain gas (triangles; Shapiro \& Benjamin 1993; Benjamin \& Shapiro 1993), turbulent mixing layers (asterisks; Slavin et al. 1993), conductive heating and evaporation of spherical and planar clouds (crosses; Boehringer \& Hartquist 1987 and Borkowski et al. 1990, respectively), cooling supernova remnant shells (plus signs; Slavin \& Cox 1993; Shelton 1998), and CIE (solid line; Sutherland \& Dopita 1993). The suite of average values from three-dimensional MHD turbulent mixing calculations of Esquivel et al. (2006) is indicated by bright shaded regions (no magnetic field) and dark shaded regions (with magnetic field). Note that the shaded regions near the center are the results of their models with radiative cooling, while the shaded regions in the upper right are those without radiative cooling. The median values in Table 7 are plotted as filled (NEI) and empty (CIE) circles and the averaged value of halo observations along 34 sightlines (Indebetouw \& Shull 2004b) is indicated by a filled star. The filled square is the result of the additional simulation mentioned in Section 4.2.

1997; Jeong et al. 2000; Ryu et al. 2000). These two effects may affect the column densities of high ions because the region for the high ions' existence is where the turbulent motion occurs. However, as the comparison of our two-dimensional hydrodynamic simulations with the three-dimensional MHD simulations of Esquivel et al. (2006) in the following section (Section 4.2) shows, including the effects of the three-dimensional geometry and magnetic field would not significantly affect the ratios between high ion column densities. The turbulent mixing model would still distinguish itself from models with other physical processes regardless of these two effects (see Figure 7). (Note that the CIE and NEI calculations of our simulations predict similar ratios and that Esquivel et al. 2006 used only the CIE calculations for the high ion column densities.)

The effects of thermal conduction for the turbulent mixing layers were discussed in Esquivel et al. (2006). Following their estimation, our model parameters lead to a similar Spitzer thermal diffusion coefficient $\kappa_{\mathrm{Sp}} \sim 10^{24} \mathrm{~cm}^{2} \mathrm{~s}^{-1}$ with $T_{\text {mixed }} \sim$ $10^{5} \mathrm{~K}$ and $n_{\text {mixed }} \sim 10^{-2} \mathrm{~cm}^{-3}$ but a larger turbulent diffusion coefficient $\kappa_{\text {turb }} \sim \frac{1}{3} v_{\text {turb }} L_{\text {inj }} \sim 10^{27} \mathrm{~cm}^{2} \mathrm{~s}^{-1}$ with $L_{\text {inj }} \sim$ $100 \mathrm{pc}$ and $v_{\text {turb }} \sim 100 \mathrm{~km} \mathrm{~s}^{-1}$, where $L_{\text {inj }}$ is the energy injection scale (approximated as the domain size) and $v_{\text {turb }}$ is the turbulent speed. In this estimation, we assume that the mixed gas has a typical temperature of $T \sim 10^{5} \mathrm{~K}$ and a density of $n \sim 10^{-2} \mathrm{~cm}^{-3}$ as in Esquivel et al. (2006). As mentioned in Esquivel et al. (2006), the larger turbulent diffusion coefficient than the Spitzer thermal diffusion coefficient implies that the heat transfer is dominated by turbulence rather than thermal diffusion which has a smaller scale. However, the small-scale diffusion process (including numerical diffusion) could affect our simulations, especially at early times. (The effect of spatial resolution that corresponds to the numerical diffusion is discussed in Section 3.2.) In our simulations as well as in the simulations of Esquivel et al. (2006), the thermal diffusion coefficients at early times when the mixing zone is very shallow are larger than the above estimated values because the gas in the initial interface between hot and cool gas has a larger temperature gradient than the gas in the interfaces between mixed and hot gas or between mixed and cool gas at later times. Therefore, both in our and their hydrodynamic simulations, the heat transfer would be faster at early times with thermal conduction included. (Note that Esquivel et al. 2006 ran both hydrodynamic and MHD simulations but did not include thermal conduction.)

\subsection{Comparison with Observations and Other Models}

The column density ratios between different ions for all of our model simulations are summarized in Table 7 . Note that in our column density calculations, we use sightline geometries that are perpendicular to the initial interface between hot and cool gas (i.e., perpendicular to the initial velocity vector of the cool gas). This choice of sightlines is the same as Slavin et al. (1993) and Esquivel et al. (2006). Along these sightlines, the measured velocities of each ion are so small that they can be directly compared with the halo observations $(\bar{v} \approx 0)$.

The column density ratios in Table 7 are plotted in the $\log [N(\mathrm{~N} \mathrm{v}) / N(\mathrm{O}$ VI $)]-\log [N(\mathrm{C}$ IV $) / N(\mathrm{O}$ VI $)]$ space for the comparison with halo observations and other models (Figure 7$)$. The averaged value from the halo observations along 34 sightlines in Indebetouw \& Shull (2004b) is indicated by a filled star in Figure 7. The median values in Table 7 are plotted as filled (NEI) and empty (CIE) circles. Error bars indicate minimum and maximum values.

The median values from our model simulations are with a factor of $\sim 5$ of the analytic turbulent mixing results of Slavin et al. (1993). The three-dimensional hydrodynamic and MHD simulations of Esquivel et al. (2006) in which radiative cooling was allowed produce similar column density ratios as our simulations although they only used CIE calculations for the column density estimations (Figure 7). Note that the bright and dark shaded regions near the center of Figure 7 represent their models that include radiative cooling, while the shaded regions in the upper right in Figure 7 represent the results of their models without any radiative cooling. (The bright and dark shaded regions indicate their models without magnetic field and with magnetic field, respectively.) The turbulent mixing phenomenon, evaluated by both analytic and numerical means yields larger $N(\mathrm{Nv}) / N(\mathrm{O}$ vI $)$ and $N(\mathrm{C}$ IV $) / N(\mathrm{O}$ vI $)$ ratios when compared with other phenomena such as radiative cooling, supernova remnants (SNRs), and conductive heating (Figure 7).

The average ion ratios predicted by our models are similar to the average from halo observations, however, our models produce more $\mathrm{CIV}$ and $\mathrm{Nv}$. From our model simulations, we find a trend that models with small velocity differences between the cool and hot gas (i.e., Model D) produce more $\mathrm{C}$ IV and we find that models with higher hot gas temperatures (i.e., Model F) produce more $\mathrm{C}$ IV and less $\mathrm{N} v$. In order to confirm this trend and to find a case closer to the observed value, we run an additional NEI simulation with an initial speed of $150 \mathrm{~km} \mathrm{~s}^{-1}$ and a hot gas temperature of $2.0 \times 10^{6} \mathrm{~K}$. The result of this additional simulation is plotted as a filled square in Figure 7 with the same convention as the other 
models of our simulations $(\log [N(\mathrm{~N} \mathrm{v}) / N(\mathrm{O} \mathrm{vI})] \approx-0.74$ and $\log [N(\mathrm{C}$ IV $) / N(\mathrm{O}$ VI $)] \approx 0.17)$. As expected, the filled square is shifted toward the star (observed value) from the data points of Model D and Model F.

It was pointed out in Indebetouw \& Shull (2004b) that the collection of halo observations exhibits a wide range of column density ratios (their Figure 4) that cannot be explained by a single type of model. However, as discussed in Section 4.1, the variations of column densities in dynamical models may explain the wide range of observed column density ratios. The error bars shown in Figure 7 are estimated only for the temporal variations after the column densities are averaged over all the sightlines at a given time. If the variations along sightlines are considered, the column density ratios estimated from our simulations are further scattered in the plot.

Finally, it is interesting to compare the results of our simulations with the ratios of high ion column densities observed with high velocities (i.e., highly ionized HVCs). For example, Collins et al. (2007) and Fox et al. (2004) measured the high ion ratios along three sightlines toward Complex $\mathrm{C}$. The combined values from their measurements are $N(\mathrm{C}$ IV $) / N(\mathrm{O}$ VI $)=0.44_{-0.06}^{+0.06}$ and $N(\mathrm{~N} \mathrm{v}) / N(\mathrm{O}$ vI $)=0.19_{-0.07}^{+0.06}$ for Mrk $279, N(\mathrm{C} \mathrm{IV}) / N(\mathrm{O} \mathrm{vI})=$ $0.40_{-0.04}^{+0.04}$ and $N(\mathrm{Nv}) / N(\mathrm{O} \mathrm{vI})<0.11$ for Mrk 876, and $N\left(\mathrm{C}_{\mathrm{IV}}\right) / N(\mathrm{O}$ vI $)=0.35_{-0.06}^{+0.05}$ and $N(\mathrm{~N} \mathrm{v}) / N\left(\mathrm{O}_{\mathrm{VI}}\right)<0.07$ for PG 1259+593. (We choose the measured values when they are available from each reference article. When the measured values are different in different articles, we choose smaller values for the measured ion ratios and smaller upper limits for $N(\mathrm{~N} \mathrm{v}) /$ $N(\mathrm{O} \mathrm{VI})$. Note that the difference between two different values are not significant so that choosing larger values would not affect the following comparison.) These measurements would be close to the average halo observations if they were plotted in Figure 7. However, the measured metallicities of carbon, nitrogen, and oxygen in Complex $\mathrm{C}$ are much lower than the solar metallicities. Fox et al. (2004) measured the abundance of metals in Complex C along the sightline toward PG 1259+593 (see Table 8 for their measured carbon, nitrogen, and oxygen abundances relative to Allen 1973). Collins et al. (2007) measured the average metallicity of Complex $\mathrm{C}$ as 0.13 solar metallicity based upon $[\mathrm{O} \mathrm{I} / \mathrm{HI}$ ] measurements along 11 sightlines toward Complex C. They also found that there is much less nitrogen in Complex $\mathrm{C}$ by factor of 0.01 to 0.07 relative to solar abundance.

When we apply different metallicities to the ion ratios calculated from our simulations, we need to shift the data points of our simulations according to Table 8 . Applying the measured abundances of metals in Complex $\mathrm{C}$ in Fox et al. (2004) to our calculations would shift all of our data points in Figure 7 by -0.35 and -0.97 along the $N(\mathrm{CIV}) / N(\mathrm{O}$ VI $)$ and $N(\mathrm{Nv}) / N(\mathrm{O} v \mathrm{I})$ axes, respectively. However, other data points from different models also need to be shifted by the same amount. This implies that our turbulent mixing model is more likely to explain the observations of highly ionized HVCs than are other physical phenomena. But, as mentioned before, the results presented in this paper pertain to sightlines that perpendicularly intersect the turbulent mixing layers and so see nearly stationary velocities of calculated column densities. In a future study, we will investigate the velocity-resolved column densities, particularly focusing on these HVC observations.

\section{SUMMARY}

We investigate the turbulent mixing layer model by running two-dimensional numerical simulations. Our simulations in- clude radiative cooling and NEI calculations. NEI calculations produce more high ions than CIE calculations: 4.6, 2.9, and 2.3 times higher column densities for $\mathrm{C}$ IV, $\mathrm{N} v$, and $\mathrm{O}$ vI, respectively, for our standard model (Model A; Tables 1 and 5). We find that in NEI calculations, both ionization and recombination for these ions are delayed, resulting in more Li-like ions $(\mathrm{C}$ IV, $\mathrm{Nv}$, and $\mathrm{O} \mathrm{vI})$ than are present in the CIE calculations (Figure 5 and Section 3.1.3). Many C IV ions, in particular, are produced even in radiatively cooled mixed gas $\left(T \leqslant 10^{4} \mathrm{~K}\right)$ at the base of the mixing zone because the recombination of $\mathrm{C}$ IV is slow in this relatively cool gas. These results are also valid in our other NEI simulations, namely, those with higher resolution (Model B) and 1/10 smaller computational domain (Model C).

By changing the model parameters such as the shear speed, the initial amplitude of the interface, and the temperature of hot gas, we study the various configurations of the turbulent mixing layer. We find that more $\mathrm{C}$ IV is produced when the shear speed is smaller (Model D) and more C IV and O VI are produced when the hot reservoir has a higher temperature (Model F). The initial amplitude does not affect the column densities significantly (Model E).

The study of various configurations shows that the column densities and corresponding ratios between the column density of different ions do not vary greatly when we vary the model parameters within the reasonable conditions for the mixing layers (a few times $10^{6} \mathrm{~K}$ for hot gas and a few hundreds $\mathrm{km} \mathrm{s}^{-1}$ for the shear speed). When the results of our simulations are plotted together with previous model calculations in $\log [N(\mathrm{~N}$ v $) / N(\mathrm{O}$ VI $)]-\log [N(\mathrm{C}$ IV $) / N(\mathrm{O}$ vI $)]$ space (Figure 7$)$, they are consistent with the analytic estimations of Slavin et al. (1993) and the three-dimensional MHD calculations of Esquivel et al. (2006). All turbulent mixing layer calculations either analytic or numerical predict more $\mathrm{CIV}$ and $\mathrm{Nv}$ than the other models (radiative cooling, cooling SNR, and conductive heating).

We compare NEI and CIE predictions for a subset of our models, finding that the CIE calculations predict smaller values of $N(\mathrm{C}$ IV $) / N(\mathrm{O}$ VI) and $N(\mathrm{~N} \mathrm{v}) / N(\mathrm{O}$ VI) (see the open circles for Models $\mathrm{A}, \mathrm{B}$, and $\mathrm{C}$ in the $\log [N(\mathrm{~N} \mathrm{v}) / N(\mathrm{O} \mathrm{vI})]-\log [N(\mathrm{C}$ IV $) /$ $N(\mathrm{O} \mathrm{vI})]$ plot, Figure 7). However, the deviation of CIE from NEI is not as large as that between different phenomenological models, especially between the turbulent mixing layer models and the other models, or as large as the variation between different sightlines or times in a single model.

Because our NEI simulations are capable of calculating the column densities of various ions along with velocity information, the velocity-resolved column densities, especially in highly ionized HVCs, can be studied by using our simulations and comparing with observations of previous studies such as Collins et al. (2007) and Fox et al. (2004) and future observations from STIS and the Cosmic Origins Spectrograph newly installed on the Hubble Space Telescope. We will investigate the velocityresolved column densities in a future study.

The FLASH code used in this work was developed in part by the DOE-supported ASC/Alliance Center for Astrophysical Thermonuclear Flashes at the University of Chicago. The simulations were performed at the Research Computing Center (RCC) of the University of Georgia. We thank the anonymous referee for his or her valuable comments on the radiative cooling and timescales. This work was supported through grant NNX09AD13G through the NASA ATPF program. 


\section{REFERENCES}

Allen, C. W. 1973, Astrophysical Quantities (London: Univ. London, Athlone Press)

Allende Prieto, C., Lambert, D. L., \& Asplund, M. 2001, ApJ, 556, L63

Allende Prieto, C., Lambert, D. L., \& Asplund, M. 2002, ApJ, 573, L137

Anders, E., \& Ebihara, M. 1982, Geochim. Cosmochim. Acta, 46, 2363

Anders, E., \& Grevesse, N. 1989, Geochim. Cosmochim. Acta, 53, 197

Asplund, M., Grevesse, N., \& Sauval, A. J. 2005, in ASP Conf. Ser. 336, Cosmic Abundances as Records of Stellar Evolution and Nucleosynthesis, ed. T. G. Barnes III \& F. N. Bash (San Francisco, CA: ASP), 25

Ballet, J., Arnaud, M., \& Rothenflug, R. 1986, A\&A, 161, 12

Bayly, B. J. 1986, Phys. Rev. Lett., 57, 2160

Begelman, M. C., \& Fabian, A. C. 1990, MNRAS, 244, 26P

Benjamin, R. A., Benson, B. A., \& Cox, D. P. 2001, ApJ, 554, L225

Benjamin, R., \& Shapiro, P. 1993, in UV and X-ray Spectroscopy of Laboratory and Astrophysical Plasmas, ed. E. H. Silver \& S. M. Kahn (Cambridge: Cambridge Univ. Press), 280

Boehringer, H., \& Hartquist, T. W. 1987, MNRAS, 228, 915

Borkowski, K. J., Balbus, S. A., \& Fristrom, C. C. 1990, ApJ, 355, 501

Bowen, D. V., et al. 2008, ApJS, 176, 59

Bregman, J. N., Fabian, A. C., Miller, E. D., \& Irwin, J. A. 2006a, ApJ, 642, 746

Bregman, J. N., Otte, B., Miller, E. D., \& Irwin, J. A. 2006b, ApJ, 642, 759

Collins, J. A., Shull, J. M., \& Giroux, M. L. 2007, ApJ, 657, 271

Cowie, L. L., Taylor, W., \& York, D. G. 1981, ApJ, 248, 528

Cox, D. P. 2005, ARA\&A, 43, 337

Craik, A. D. D., \& Criminale, W. O. 1986, Proc. R. Soc. A, 406, 13

Danforth, C. W., \& Blair, W. P. 2006, ApJ, 646, 205

Dixon, W. V. D., Davidsen, A. F., \& Ferguson, H. C. 1996, ApJ, 465, 288

Dixon, W. V. D., Sankrit, R., \& Otte, B. 2006, ApJ, 647, 328

Edgar, R. J., \& Chevalier, R. A. 1986, ApJ, 310, L27

Esquivel, A., Benjamin, R. A., Lazarian, A., Cho, J., \& Leitner, S. N. 2006, ApJ, 648, 1043

Ferriere, K. 1998, ApJ, 497, 759

Fox, A. J., Ledoux, C., Petitjean, P., \& Srianand, R. 2007, A\&A, 473, 791

Fox, A. J., Ledoux, C., Vreeswijk, P. M., Smette, A., \& Jaunsen, A. O. 2008, A\&A, 491, 189

Fox, A. J., Prochaska, J. X., Ledoux, C., Petitjean, P., Wolfe, A. M., \& Srianand, R. 2009, A\&A, 503, 731

Fox, A. J., Savage, B. D., Wakker, B. P., Richter, P., Sembach, K. R., \& Tripp, T. M. 2004, ApJ, 602, 738

Fox, A. J., Wakker, B. P., Savage, B. D., Tripp, T. M., Sembach, K. R., \& Bland-Hawthorn, J. 2005, ApJ, 630, 332

Frank, A., Jones, T. W., Ryu, D., \& Gaalaas, J. B. 1996, ApJ, 460, 777

Fryxell, B., et al. 2000, ApJS, 131, 273

Ganguly, R., Sembach, K. R., Tripp, T. M., \& Savage, B. D. 2005, ApJS, 157, 251

Ganguly, R., Sembach, K. R., Tripp, T. M., Savage, B. D., \& Wakker, B. P. 2006, ApJ, 645, 868

Gnat, O., \& Sternberg, A. 2007, ApJS, 168, 213

Gnat, O., Sternberg, A., \& McKee, C. F. 2010, ApJ, in press (arXiv:1002.1309G)

Grevesse, N. 1984, Phys. Scr. T, 8, 49
Grevesse, N., Noels, A., \& Sauval, A. J. 1996, in ASP Conf. Ser. 99, Cosmic Abundances, ed. S. S. Holt \& G. Sonneborn (San Francisco, CA: ASP), 117

Holweger, H. 2001, in AIP Conf. Ser. 598, Joint SOHO/ACE Workshop on Solar and Galactic Composition, ed. R. F. Wimmer-Schweingruber (Melville, NW: AIP), 23

Hussain, A. K. M. F. 1984, in Turbulence and Chaotic Phenomena in Fluids, ed. T. Tatsumi (New York: Elsevier), 453

Indebetouw, R., \& Shull, J. M. 2004a, ApJ, 605, 205

Indebetouw, R., \& Shull, J. M. 2004b, ApJ, 607, 309

Jenkins, E. B. 1978a, ApJ, 219, 845

Jenkins, E. B. 1978b, ApJ, 220, 107

Jenkins, E. B. 2004, Ap\&SS, 289, 215

Jeong, H., Ryu, D., Jones, T. W., \& Frank, A. 2000, ApJ, 529, 536

Jones, T. W., Gaalaas, J. B., Ryu, D., \& Frank, A. 1997, ApJ, 482, 230

Korpela, E. J., et al. 2006, ApJ, 644, L163

Lehner, N., \& Howk, J. C. 2007, MNRAS, 377, 687

Oegerle, W. R., Cowie, L., Davidsen, A., Hu, E., Hutchings, J., Murphy, E., Sembach, K., \& Woodgate, B. 2001, ApJ, 560, 187

Otte, B., \& Dixon, W. V. D. 2006, ApJ, 647, 312

Prochaska, J. X., Dessauges-Zavadsky, M., Ramirez-Ruiz, E., \& Chen, H.-W. 2008, ApJ, 685, 344

Raymond, J. C., \& Smith, B. W. 1977, ApJS, 35, 419

Ryu, D., Jones, T. W., \& Frank, A. 2000, ApJ, 545, 475

Sallmen, S. M., Korpela, E. J., \& Yamashita, H. 2008, ApJ, 681, 1310

Sankrit, R., Blair, W. P., \& Raymond, J. C. 2004, AJ, 128, 1615

Savage, B. D., \& Massa, D. 1987, ApJ, 314, 380

Savage, B. D., Meade, M. R., \& Sembach, K. R. 2001a, ApJS, 136, 631

Savage, B. D., \& Sembach, K. R. 1994, ApJ, 434, 145

Savage, B. D., Sembach, K. R., \& Howk, J. C. 2001b, ApJ, 547, 907

Savage, B. D., Sembach, K. R., \& Lu, L. 1997, AJ, 113, 2158

Savage, B. D., et al. 2003, ApJS, 146, 125

Sembach, K. R., \& Savage, B. D. 1992, ApJS, 83, 147

Sembach, K. R., Savage, B. D., \& Tripp, T. M. 1997, ApJ, 480, 216

Sembach, K. R., et al. 2003, ApJS, 146, 165

Shapiro, P. R., \& Benjamin, R. A. 1993, in Star Formation, Galaxies and the Interstellar Medium, ed. J. Franco, F. Ferrini, \& G. Tenorio-Tagle (Cambridge: Cambridge Univ. Press), 275

Shelton, R. L. 1998, ApJ, 504, 785

Shelton, R. L., Sallmen, S. M., \& Jenkins, E. B. 2007, ApJ, 659, 365

Shelton, R. L., et al. 2001, ApJ, 560, 730

Shull, J. M., \& Slavin, J. D. 1994, ApJ, 427, 784

Slavin, J. D., \& Cox, D. P. 1992, ApJ, 392, 131

Slavin, J. D., \& Cox, D. P. 1993, ApJ, 417, 187

Slavin, J. D., Shull, J. M., \& Begelman, M. C. 1993, ApJ, 407, 83

Sterling, N. C., Savage, B. D., Richter, P., Fabian, D., \& Sembach, K. R. 2002, ApJ, 567, 354

Sutherland, R. S., \& Dopita, M. A. 1993, ApJS, 88, 253

Thom, C., Peek, J. E. G., Putman, M. E., Heiles, C., Peek, K. M. G., \& Wilhelm, R. 2008, ApJ, 684, 364

Tripp, T. M., Sembach, K. R., Bowen, D. V., Savage, B. D., Jenkins, E. B., Lehner, N., \& Richter, P. 2008, ApJS, 177, 39

Welsh, B. Y., et al. 2007, A\&A, 472, 509

Zsargó, J., Sembach, K. R., Howk, J. C., \& Savage, B. D. 2003, ApJ, 586, 1019 